\title{
Ferromagnetic resonance spectroscopy for assessment of magnetic anisotropy and magnetostatic interactions: a case study of mutant magnetotactic bacteria
}

\author{
Robert E. Kopp ${ }^{1 *}$, Cody Z. Nash ${ }^{1}$, Atsuko Kobayashi ${ }^{2}$, Benjamin P. Weiss ${ }^{3}$, Dennis
} A. Bazylinski ${ }^{4}$, and Joseph L. Kirschvink ${ }^{1}$

Received 25 May 2006; revised 28 September 2006; accepted 25 October 2006.

Published 28 December 2006 in the Journal of Geophysical Research - Solid Earth, special section on Rock Magnetism: Fundamentals and Frontiers.

Ferromagnetic resonance spectroscopy (FMR) can be used to measure the effective magnetic field within a sample, including the contributions of both magnetic anisotropy and magnetostatic interactions. One particular use is in the detection of magnetite produced by magnetotactic bacteria. These bacteria produce single-domain particles with narrow size and shape distributions that are often elongated and generally arranged in chains. All of these features are detectable through FMR. Here, we examine their effects on the FMR spectra of magnetotactic bacteria strains MV-1 (which produces chains of elongate magnetite crystals), AMB-1 (which produces chains of nearly equidimensional magnetite crystals), and two novel mutants of AMB-1: mnm 13 (which produces isolated, elongate crystals), and $\mathrm{mnm} 18$ (which produces nearly equidimensional crystals that are usually isolated). Comparison of their FMR spectra indicates that the positive magnetic anisotropy indicated by the spectra of almost all magnetotactic bacteria is a product of chain alignment and particle elongation. We also find correlations between FMR properties and magnetic measurements of coercivity and magnetostatic interactions. FMR thus provides a rapid method for assessing the magnetic properties of assemblages of particles, with applications including screening for samples likely to contain bacterial magnetofossils.

Citation: R. E. Kopp, C. Z. Nash, A. Kobayashi, B. P. Weiss, D. A. Bazylinski, and J. L. Kirschvink (2006). Ferromagnetic resonance spectroscopy for assessment of magnetic anisotropy and magnetostatic interactions: A case study of mutant magnetotactic bacteria. J. Geophys. Res. B 111, B12S25. doi:10.1029/2006JB004529

\footnotetext{
${ }^{1}$ Division of Geological and Planetary Sciences, California Institute of Technology, Pasadena, CA 91125, USA

${ }^{2}$ Photonics Research Institute, National Institute of Advanced Industrial Science and Technology, 1-8-31 Midorigaoka, Ikeda, Osaka 563-8577, Japan

${ }^{3}$ Department of Earth, Atmospheric, and Planetary Sciences, Massachusetts Institute of Technology, Cambridge, MA 02139 , USA

${ }^{4}$ Department of Biochemistry, Biophysics, and Molecular Biology, Iowa State University, Ames, IA 50011, USA

* Corresponding author. Caltech 170-25, Pasadena, CA 91125, USA. E-mail: rkopp at caltech.edu, Telephone: +1 626-395-2949, Fax: +1 626-568-0935.
}

Index terms: 1505 biogenic magnetic minerals, 1518 magnetic fabrics and anisotropy, 1540 rock and mineral magnetism, 0419 biomineralization, 0465 microbiology.

Key words: ferromagnetic resonance spectroscopy, magnetotactic bacteria, magnetofossils, magnetic anisotropy, magnetostatic interactions, rock magnetism

An edited version of this paper was published by AGU. Copyright (C) 2006 American Geophysical Union. 


\section{Introduction}

Ferromagnetic resonance spectroscopy (FMR), a form of electron spin resonance spectroscopy, can serve as a rapid technique for assessing the magnetic anisotropy of and magnetostatic interactions between individual particles in a polycrystalline sample. It is based upon the Zeeman effect, which is the splitting between electron spin energy levels that occurs in the presence of a magnetic field. The Zeeman effect allows a ground-state electron to absorb a photon with energy equal to the splitting between the energy states. In a magnetic material, magnetic anisotropy (whether magnetocrystalline, shape, or stress-induced) and interparticle interactions contribute to the energy of the particles within a sample and thereby alter the resonance energy. As a result, FMR can be used to probe these parameters [Griscom, 1974; Griscom, 1981; Kittel, 1948; Kopp, et al., 2006; Schlömann, 1958; Weiss, et al., 2004].

Techniques for measuring anisotropy and magnetostatic interactions have a number of applications in the field of rock magnetism. The example on which we will focus here is the identification of magnetite produced by magnetotactic bacteria, a topic of great interest for understanding the magnetization of sediments. Fossil magnetotactic bacteria may also serve as paleoenvironmental indicators of both strong magnetic fields and local redox gradients [Chang and Kirschvink, 1989; Kirschvink and Chang, 1984]. These bacteria are a phylogenetically diverse group that biomineralize intracellular crystals of magnetic minerals (magnetite or greigite) which orient the bacteria passively in the geomagnetic field. Natural selection has led these bacteria to optimize the magnetic moment produced for the amount of iron used. Among the traits present in magnetite produced by many magnetotactic bacteria are a narrow distribution of particle sizes within the single-domain stability field, particle elongation, and the arrangement of particles in chains [Thomas-Keprta, et al., 2000]. The biophysical problem of keeping strongly magnetic particles aligned in a chain may also have driven the evolution of a variety of cytoskeletal supporting mechanisms, including an intracellular 'sheath' [Kobayashi, et al., 2006], actin-like cytoskeletal filaments [Scheffel, et al., 2006] and/or direct attachments to the periplasmic membrane [Komeili, et al., 2006].

The adaptive traits possessed by these biogenic magnetic particles at a microscopic level generate distinct magnetic properties that are identifiable with macroscopic techniques. The particles' narrow distribution within the single domain size range is typically observed in analyses of coercivity spectra, including the measurement of the acquisition of isothermal remanent magnetization and the demagnetization of remanent magnetizations [Chang, et al., 1989; Pan, et al., 2005]. [Egli, 2004] used the unmixing of coercivity spectra to determine the biogenic contribution to lacustrine sedimentary magnetization.

Anhysteretic susceptibility, which provides a qualitative measure of inverse interaction strength when comparing single domain particles of similar volumes [Dunlop, et al., 1990; Egli and Lowrie, 2002], has also been used to distinguish bacterial magnetite chains from abiogenic magnetite [Kopp, et al., 2006; Moskowitz, et al., 1993]. Anhysteretic magnetization is acquired by the application of a small biasing field in the presence of a decaying alternating field. In the absence of thermal effects, non-interacting single-domain particles would have infinite anhysteretic susceptibility; they should become magnetized in the direction of the biasing field as soon as the alternating field decreases below their microcoercivity [Dunlop and Özdemir, 1997; Egli and Lowrie, 2002]. In fact, thermal effects cause more elongate and smaller particles to have lower ARM susceptibility than less elongate or larger single-domain particles [Egli and Lowrie, 
2002]. The shielding effects of magnetostatic interactions operating in three dimensions also lower anhysteretic susceptibility.

In many strains of magnetotactic bacteria, however, linear magnetostatic interactions cause an entire chain of particles to behave in a magnetically coherent fashion [Hanzlik, et al., 2002; Penninga, et al., 1995]. Intact cells of magnetotactic bacteria therefore have low threedimensional magnetostatic interactions and thus relatively high anhysteretic susceptibility, so high anhysteretic remanent magnetization (ARM)/isothermal remanent magnetization (IRM) ratios are characteristic of the presence of magnetite chains. Collapsed magnetosome chains, with stronger three-dimensional magnetostatic interactions, have lower ARM/IRM ratios.

Another test that is indicative of the presence of chains is the delta-delta test of [Moskowitz, et al., 1993], which uses the ratio of magnetization lost upon warming through the $\sim 90-120 \mathrm{~K}$ Verwey transition in saturated samples that have been cooled in a strong field to the magnetization lost after cooling in zero field. While previous data indicate that this test does identify chains of biogenic magnetite [Moskowitz, et al., 1993; Weiss, et al., 2004], it is susceptible to false negatives and the underlying physical mechanisms are incompletely understood [Carter-Stiglitz, et al., 2004].

Ferromagnetic resonance spectroscopy is capable of rapidly distinguishing biogenic magnetite chains based on three traits: (i) a narrow range of particle size, shape, and arrangement, (ii) chain structure, and (iii) particle elongation [Kopp, et al., 2006; Weiss, et al., 2004]. Samples with narrow distributions of size, shape, and arrangement have narrow FMR peaks. Chain structure and particle elongation produce positive uniaxial anisotropy, which can be distinguished from the negative cubic magnetocrystalline anisotropy that dominates isolated, equidimensional magnetite.

Bacterial mutagenesis is a central technique in molecular microbiology. By disabling regions of the genome, it probes the roles of different genes in the production of a phenotype. Our attempts to understand the molecular mechanism of magnetite biosynthesis (which will be described in a follow-up paper by Nash et al.) led us to create mutant strains of the magnetotactic bacterium Magnetospirillum magneticum strain AMB-1, whose wild-type creates chains of almost equidimensional cubo-octahedral crystals. Two of these mutants produce crystals that are usually isolated and are either approximately equidimensional (mutant $\mathrm{mnm} 18$ ) or elongate (mutant mnm13). We used these mutants, along with cells of wild-type AMB-1 and the magnetotactic marine vibrio MV-1, which produces chains of elongate hexa-octahedral crystals, to investigate the contributions of magnetic anisotropy and magnetostatic interactions to ferromagnetic resonance spectra. These different strains allow us for the first time to separate directly the effects of chain structure on FMR and rock magnetic properties from those of single crystal traits.

\section{Methods}

\section{Mutagenesis}

To generate the mutants, transposon mutagenesis was performed on AMB-1 following previously described procedures [Komeili, et al., 2004]. Mutants were grown up on plates, and single colonies were then picked and grown up in 96-well plates in sealed jars with $2 \%$ oxygen/98\% nitrogen atmospheres. After 3-5 days of growth, weakly magnetic and nonmagnetic mutants were identified by placing the plates on an array of magnets. Mutants that were 
not drawn towards the side of the well were subcultured for further analysis. For mutant mnm13, sequencing of genomic DNA indicated that an interruption by the introduced transposon occurred in a gene encoding for a hypothetical protein. For mnm 18, sequencing indicated that the interruption occurred in a pyruvate/ferredoxin oxidoreductase gene. Time course experiments indicate that $m n m 18$ is a growth defective mutant that takes 1-2 days longer to reach stationary phase than the wild-type.

\section{Growth conditions and lysis}

Cells of strain MV-1 were grown anaerobically with nitrous oxide as the terminal electron acceptor under heterotrophic conditions as previously described [Dean and Bazylinski, 1999]. Cells were harvested at early stationary phase, at a cell density of about $1.5 \times 10^{9}$ cells $/ \mathrm{mL}$, by centrifugation at $5,000 \mathrm{xg}$ at $4^{\circ} \mathrm{C}$ for $10 \mathrm{~min}$ and then resuspended in ice-cold artificial seawater containing $20 \mathrm{mM}$ Tris- $\mathrm{HCl}$ at $\mathrm{pH}$ 7.0. Cells were recentrifuged and the resultant pellet of cells was frozen and shipped on dry ice to Caltech, where it was thawed. A fraction of the cell mass was resuspended in Tris buffer, from which point it was subject to the same treatments as AMB-1.

Two liters each of AMB-1 wild-type and mutants mnm13 and mnm18 were grown up to early stationary phase, at a cell density of about $10^{8}$ cells $/ \mathrm{mL}$, using standard culture conditions [Komeili, et al., 2004]. The cultures were divided into thirds (A1, A2, and A3 for the wild-type; $\mathrm{B} 1, \mathrm{~B} 2$, and B3 for mnm 13; C1, C2, and C3 for mnm 18; V1, V2, and V3 for MV-1), spun down, and resuspended in $\sim 5 \mathrm{~mL} 100 \mathrm{mM}$ Tris buffer at $\mathrm{pH}$ 7. Five $\mu \mathrm{L}$ of $\beta$-mercaptoethanol and $\sim 250$ mg of sodium dodecyl sulfate (SDS) were added to subsamples A3, B3, C3, and V3. Subsamples A2, A3, B2, B3, C2, C3, V2, and V3 were then subjected to ultrasonication with a Fisher

Scientific Sonic Dismembrator 550 for about six minutes, with pulses of 0.5 seconds alternating with pauses of equal duration. Ultrasonication should destroy cell membranes while leaving magnetosome membranes intact. SDS treatment should destroy both cell membranes and magnetosome membranes, thereby freeing the magnetite particles from organic structures.

The samples were then spun down, frozen, and freeze-dried. In a set of experiments analogous to the dilution experiments described in [Kopp, et al., 2006], V2 was diluted at $\sim 1$ part per thousand in sucrose. It was initially measured as sample V2a, then was diluted by mixing with a mortar and pestle for 4 minutes to form subsample V2b. Sample V3 was similarly diluted at $\sim 1$ part per thousand as sample V3a, diluted by mixing for 1 minute to form sample V3b, and then mixed for four additional minutes to form sample V3c.

\section{Electron microscopy}

Specimens were dispersed on hydrophilic copper TEM grids, air-dried, and made electrically conductive by coating with a thin carbon film using conventional methods. The grids were inserted into a beryllium TEM specimen holder for EDS analysis. TEM and HAADF/STEM images were obtained with a Tecnai G2 F20 Twin (FEI, Holland), operating at 200kV and equipped with Gatan energy filter GIF2001 and HAADF/STEM detecting unit. The HAADF/STEM/EDS analysis was performed by an EDX detecting unit (EDAX, Inc.). Histograms of particle size/shape distributions were made by measuring the maximum length and widths of magnetite crystals visible in the TEM images in a similar fashion to that of [Kirschvink and Lowenstam, 1979] and [Devouard, et al., 1998]. Due to the sharp decay in field 
strength with distance $\left(1 / \mathrm{r}^{3}\right)$, particles were grouped into chains if they were positioned within less than one grain diameter from an adjacent crystal.

\section{Ferromagnetic resonance spectra}

Ferromagnetic resonance spectra were acquired using an X-band Bruker ESP 300E EPR Spectrometer housed at Caltech. Except for particularly strong samples (V3a, V3b, and V3c), microwave power was set at $640 \mu \mathrm{W}$ and spectra were integrated over three sweeps of the applied field from 0 to $600 \mathrm{mT}$. For strong samples, power was set at $64 \mu \mathrm{W}$ and only one spectrum was acquired. To summarize spectral characteristics, we use the empirical parameters developed by [Weiss, et al., 2004] and [Kopp, et al., 2006]: $g_{\text {eff, }} A, \Delta B_{\mathrm{FWHM}}$, and $\alpha$. The effective $g$-factor, $g_{\text {eff, }}$ is the $g$-factor associated with maximum absorption which is given by $g_{\text {eff }}=$

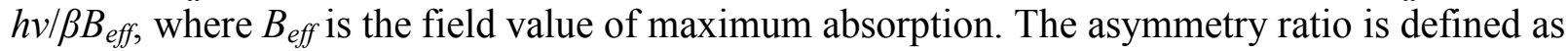
$A=\Delta B_{\text {high }} / \Delta B_{\text {low }}$, where $\Delta B_{\text {high }}=B_{\text {high }}-B_{\text {eff, }} \Delta B_{\text {low }}=B_{\text {eff }}-B_{\text {low }}$, and $B_{\text {high }}$ and $B_{\text {low }}$ are the fields of half maximum absorption at low-field and high-field sides of the absorption peak, respectively. The full width at half maximum, $\Delta B_{F W H M}$, is defined as $\Delta B_{F W H M}=B_{\text {high }}+B_{\text {low }}$. Although all these parameters are derived from the integrated absorption spectrum, FMR spectra are generally displayed as derivative spectra, which magnify fine detail. The empirical parameter $\alpha$, which serves as a proxy for the linewidth of symmetric Gaussian broadening caused by factors including heterogeneity of particle size, shape, and arrangement, is defined as $\alpha=0.17 A+9.8 \times$ $10^{-4} \Delta B_{F W H M} / \mathrm{mT}$.

The empirical parameters defined above differ from the physical parameters that control the spectral shape $\left(g, B_{a n}, K_{2} / K_{1}\right.$, and $\sigma$ ) and which we estimate using the models discussed in the Models section below. The MATLAB routines used for data analysis and fitting are available as Auxiliary Material.

\section{Room-temperature remanent magnetization experiments}

Room temperature remanent magnetization experiments were performed using a $2 \mathrm{G}$ Enterprises Superconducting Rock Magnetometer housing in a magnetically-shielded room at Caltech and equipped with in-line coils for degaussing, DC pulsing, and applying weak DC biasing fields. Starting with an AF-demagnetized sample, anhysteretic remanent magnetization (ARM) was acquired in a $100 \mathrm{mT}$ alternating field (AF) and a DC biasing field that was raised in $0.05 \mathrm{mT}$ steps to $1 \mathrm{mT}$. The ARM was then removed by step-wise AF demagnetization up to $250 \mathrm{mT}$ in logarithmically-spaced steps (where the steps were multiples of $10^{0.1} \mathrm{mT}$ ). The sample was then imparted an isothermal remanent magnetization (IRM) by pulsing with a $100 \mathrm{mT}$ field. This IRM was then removed by stepwise AF demagnetization. Finally, an IRM was imparted stepwise in logarithmically-spaced steps up to $980 \mathrm{mT}$ and then removed by AF demagnetization.

To produce coercivity spectra from the stepwise AF and IRM curves, we took the derivative of the curves with respect to the log of the applied field and smoothed the curves with a running average. We report the following parameters: the coercivity of remanence $H_{c r}$, the Cisowski crossover $R$ value, the median acquisition field of IRM (MAF IRM), the median destructive fields of IRM ( $\left.\mathrm{MDF}_{\mathrm{IRM}}\right)$ and ARM (MDF $\left.\mathrm{ARM}\right)$, and the ARM ratio $k_{A R M} / \mathrm{IRM}$.

The parameters $H_{c r}$ and $R$ are determined from the IRM stepwise acquisition and demagnetization curves. $H_{c r}$ is the field value at which the two magnetization curves cross, and $R$ is the ratio of magnetization to SIRM at that field [Cisowski, 1981]. For non-interacting single 
domain particles (or magnetically coherent chains of particles that do not interact with other chains), $R=0.5$, while decreasing values indicate increasing magnetostatic interactions. The median acquisition and destructive fields are defined as the fields required to yield half of the maximum remanent magnetization, where the IRM value is taken from the stepwise IRM curve and the ARM value is taken from the ARM demagnetization curve. We report ARM susceptibility as $k_{A R M} / I R M$, the ARM acquired per $\mathrm{A} / \mathrm{m}$ of biasing field (as measured in a biasing field of $0.1 \mathrm{mT}(79.6 \mathrm{~A} / \mathrm{m})$ and an alternating field of $100 \mathrm{mT})$, normalized to the IRM acquired in a field of $100 \mathrm{mT}$.

\section{Low temperature rock magnetic experiments}

Low temperature rock magnetic experiments were performed using a Quantum Design Magnetic Properties Measurement System (MPMS) housed in the Molecular Materials Resource Center of the Beckman Institute at Caltech. Following the procedure of [Moskowitz, et al., 1993], field cooled and zero-field cooled curves were acquired by cooling the sample either in a $3 \mathrm{~T}$ field or in zero field to $5 \mathrm{~K}$, respectively, followed by pulsing with a $3 \mathrm{~T}$ field and then measuring the remanence magnetization during warming to room temperature in zero field. Lowtemperature cycling curves when then acquired by pulsing the sample with a $3 \mathrm{~T}$ field at room temperature and then measuring the remanent magnetization as the sample was cooled to $10 \mathrm{~K}$ and then warmed to room temperature.

The results of the low-temperature experiments are reported as the parameters $\delta_{Z F C}, \delta_{F C}$, and $f_{L T C}$. The parameters $\delta=\left(J_{80 \mathrm{~K}}-J_{150 \mathrm{~K}}\right) / J_{80 \mathrm{~K}}$ were assessed for the zero-field cooled and field cooled curves respectively, where $J_{80 \mathrm{~K}}$ and $J_{150 \mathrm{~K}}$ are the moments measured at $80 \mathrm{~K}$ and $150 \mathrm{~K}$, respectively. A ratio $\delta_{F C} / \delta_{Z F C}>2.0$ passes the Moskowitz test and is considered to be an indicator of the presence of magnetosome chains, although partial oxidation and mixing can cause intact chains to fail the test [Moskowitz, et al., 1993; Weiss, et al., 2004]. Magnetization retained through low temperature cycling is expressed as the memory parameter $f_{\mathrm{LTC}}=J_{\mathrm{LTC}} / J_{0}$, where $J_{0}$ and $J_{\text {LTC }}$ are respectively the room-temperature magnetization measured before and after cycling the samples to $10 \mathrm{~K}$.

\section{Models}

The models used to fit FMR spectra in this paper are a generalization of prior models [Griscom, 1974; Griscom, 1981; Kopp, et al., 2006]. They derive from the resonance condition (equation 7 of [Smit and Beljers, 1955]):

$$
\left(\frac{h v}{g \beta}\right)^{2}=\frac{1}{M_{s}^{2} \sin ^{2} \vartheta}\left(\frac{\partial^{2} G}{\partial \vartheta^{2}} \frac{\partial^{2} G}{\partial \varphi^{2}}-\frac{\partial^{2} G}{\partial \vartheta \partial \varphi}\right)
$$

where $h v$ is the energy of the microwave photons, $g$ is the spectroscopic $g$-factor of an isolated particle with all anisotropy effects removed, $\beta$ is the Bohr magneton $\left(9.37 \times 10^{-24} \mathrm{Am}^{2}\right), M_{s}$ is the saturation magnetization, $G$ is the free energy of the system, and $\vartheta$ and $\varphi$ are the polar coordinates of the magnetization vector in its minimum energy orientation.

Neglecting thermal energy, which is isotropic and therefore does not appear in equation (1), the free energy $G$ of a system composed of non-interacting, single domain particles can be 
written as a sum of the magnetostatic energy $\mathbf{- M} \cdot \mathbf{B}$ and the anisotropy energy. When the reference frame is defined such that the anisotropy axis is directed along $(\vartheta, \varphi)=(0,0)$ as shown in Figure 1, the free energy is given by

$$
G=M_{s}\left\{-B_{a p p}[\sin \vartheta \sin \theta \cos (\phi-\varphi)+\cos \vartheta \cos \theta]+\frac{1}{2} B_{a n} F(\vartheta, \varphi)\right\}
$$

where $(\theta, \phi)$ are the polar coordinates of the experimentally applied field $B_{a p p}$ with respect to the anisotropy axis, $B_{a n}$ is an effective anisotropy field, and $F(\vartheta, \varphi)$ is a geometric factor expressing the variation of the anisotropy energy as a function of the direction of the magnetization vector.

$B_{a n}$ and $F$ vary depending on the source of the anisotropy. For magnetocrystalline anisotropy, $B_{a n}$ is $2 K_{1} / M_{\mathrm{s}}$, where $K_{1}$ is the first-order anisotropy constant (generally written as $K_{1}^{\prime}$ for uniaxial anisotropy). For uniaxial shape anisotropy, $B_{a n}$ is $\mu_{0} M_{s} \Delta N$, where $\mu_{0}$ is the magnetic permeability of free space $\left(4 \pi \times 10^{-7} \mathrm{~N} / \mathrm{A}^{2}\right)$ and $\Delta N$ is the difference between the demagnetization factors $N_{/ /}$and $N_{\perp}$ parallel and perpendicular to the elongate axis. For uniaxial anisotropy, regardless of the source,

$$
F(\vartheta)=\sin ^{2} \vartheta+\frac{K_{2}^{\prime}}{K_{1}^{\prime}} \sin ^{4} \vartheta
$$

while for cubic anisotropy,

$$
F(\vartheta, \varphi)=\sin ^{4} \vartheta \sin ^{2} \varphi \cos ^{2} \varphi+\sin ^{2} \vartheta \cos ^{2} \vartheta+\frac{K_{2}}{K_{1}} \sin ^{4} \vartheta \cos ^{2} \vartheta \sin ^{2} \varphi \cos ^{2} \varphi
$$

where $K_{2}$ is the second-order anisotropy constant (generally written as $K_{2}^{\prime}$ for uniaxial anisotropy) [Dunlop and Özdemir, 1997].

By using a first-order approximation to calculate the equilibrium orientation of the magnetization vector and considering only terms that are first order in $B_{a n} / B_{\text {res }}$, we arrive at equation A.3 of [Schlömann, 1958]:

$$
\left(\frac{h v}{g \beta B_{r e s}}\right)^{2}=1+\frac{B_{a n}}{2 B_{r e s}} a
$$

where $B_{r e s}$ is the applied field at which a particle in an arbitrary orientation achieves resonance and

$$
a=\frac{\partial^{2} F}{\partial \vartheta^{2}}+\frac{\partial^{2} F}{\partial \varphi^{2}} \cdot \frac{1}{\sin ^{2} \theta}+\frac{\partial F}{\partial \vartheta} \cot \theta
$$

Solving the quadratic expression in equation (5) yields an expression for $B_{\text {res }}$ as a function of orientation: 


$$
B_{r e s}=\sqrt{\left(\frac{h v}{g \beta}\right)^{2}+\left(\frac{a B_{a n}}{4}\right)^{2}}-\frac{a B_{a n}}{4}
$$

For uniaxial anisotropy,

$$
a_{\text {uniaxial }}=6 \cos ^{2} \theta-2+\frac{K_{2}^{\prime}}{K_{1}^{\prime}}\left(16 \cos ^{2} \theta \sin ^{2} \theta-4 \sin ^{4} \theta\right)
$$

while for cubic anisotropy,

$$
a_{\text {cubic }}=4\left(\begin{array}{l}
1-5\left(\cos ^{2} \theta \sin ^{2} \theta+\sin ^{4} \theta \sin ^{2} \phi \cos ^{2} \phi\right) \\
+\frac{K_{2}}{2 K_{1}}\left(\begin{array}{l}
\cos ^{2} \theta \sin ^{2} \theta+\sin ^{4} \theta \sin ^{2} \phi \cos ^{2} \phi \\
-21 \sin ^{4} \theta \cos ^{2} \theta \sin ^{2} \phi \cos ^{2} \phi
\end{array}\right)
\end{array}\right)
$$

When the second-order anisotropy terms in equation (9) and the second term under the radical in equation (7) are ignored, the resonance conditions thus computed are identical to those of [Griscom, 1974]. When only the second term under the radical is ignored, the cubic anisotropy condition is identical to that of [Griscom, 1981], except that in equation (9) we drop the thirdorder anisotropy term in $K_{3}$ introduced in that paper. The resonance conditions we have derived are strictly correct to first order in terms of $B_{a n} / B_{\text {res }}$ for dilute powders of single-domain particles.

To compute the powder absorption spectrum at $B_{a p p}$, we apply a Gaussian broadening function of linewidth $\sigma$ and numerically integrate the spectra over all solid angles:

$$
A\left(B_{a p p}\right)=\int_{\theta=0}^{\pi / 2} \int_{\phi=0}^{2 \pi} \frac{\exp \left(-\left(B_{a p p}-B_{r e s}(\theta, \phi)\right)^{2} / 2 \sigma^{2}\right)}{\sqrt{2 \pi} \sigma} d \phi \sin \theta d \theta
$$

The Gaussian broadening incorporates a number of physical effects, including those associated with heterogeneity of size, shape, arrangement, and composition within the sample population. To reflect the physics more accurately, the spectroscopic $g$-factor and the anisotropy parameters ought to have population distributions associated with them individually. However, attempting to fit experimental spectra to a model that employed population distributions for each of these terms would almost always be a problem without a unique solution.

We fit measured spectra to simulated spectra using non-linear least squares fitting. For each magnetic component included, the models have four parameters that can be adjusted to fit the spectra: $g, B_{a n}, K_{2} / K_{1}$, and $\sigma$. When appropriate, the spectra can be fit to two components, in which case $K_{2} / K_{1}$ is set to zero for both components in order to limit the number of additional degrees of freedom introduced.

For most of the samples, we attempted fits with both cubic and uniaxial models, as well as models combining two uniaxial components, two cubic components, or a uniaxial component and a cubic component. Except when $\left|B_{a n}\right|>>\sigma$, substituting a cubic component for a uniaxial component did not significantly improve or degrade the goodness of the fit. We suspect this is because the Gaussian broadening conceals the underlying physics in a fashion that makes it 
difficult to discriminate between samples best fit with a cubic component and those best fit with a uniaxial component.

The substitution often had only slight effects on the fitted parameters as well, but sometimes did vary the parameters outside the confidence intervals on the uniaxial fits. Because the anisotropy field expected from the cubic magnetocrystalline anisotropy of stoichiometric magnetite $\left(K_{1}=-1.35 \times 10^{4} \mathrm{~J} / \mathrm{m}^{3}, M_{s}=480 \mathrm{kA} / \mathrm{m}\right)$ is about $-56 \mathrm{mT}$ [Dunlop and Özdemir, 1997], we report the fitted parameters using cubic anisotropy for components with $B_{a n}$ between approximately -56 and $0 \mathrm{mT}$. (The dominant components of samples $\mathrm{C} 1$ and $\mathrm{C} 2$ are the only components that fit this criterion). The underlying physics in fact reflects neither purely uniaxial nor purely cubic anisotropy, but a more complicated combination of approximately uniaxial shape anisotropy and cubic magnetocrystalline anisotropy, which would be reflected in a more complete form of equation (2). Some of the Gaussian broadening likely results from our simplified treatment of the anisotropy.

The decision as to whether to represent a measured spectrum with a one-component spectrum or the sum of two model spectra was made heuristically, based upon the level of the improvement of fit when a second spectrum was added, how physically realistic the two spectra identified by the fitting routine are, and the size of the confidence intervals around fit parameters. In interpreting the models, it is important to remember that, if properly modeled, multiple components in a fit reflect multiple end-members mixed together (e.g. isolated particles and particles in chains, or particles in chains and particles in clumps); they do not reflect multiple aspects of the anisotropy of a single end-member.

\section{Results}

\section{Electron microscopy}

Consistent with [Devouard, et al., 1998], our TEM images indicate that MV-1 produces chains of magnetite crystals with a mean single crystal length of $\sim 75 \mathrm{~nm}$ and a mean length-towidth ratio of $\sim 1.8$ (Figure 2 ). The untreated cells of MV-1 we measured experienced some chain collapse, perhaps due to the freezing of the sample. As can be seen in Figure 2a, some chains collapsed into zero stray field loop configurations, while in other chains some of the particles have fallen into side-by-side arrangements. About ten percent of the crystals appear sufficiently separated from other crystals to be magnetically isolated. Collapse features are greatly enhanced by ultrasonication (Fig. 2b). Few of the chains in the ultrasonicated sample V2 are unaffected; most are bent or interwoven with other chains. Only a small number of crystals are magnetically isolated. Treatment with SDS (sample V3) led to near complete collapse of chains into clumps (Fig. 2c).

There appears to be a greater tendency for chain collapse to occur in strain MV-1 than in M. magnetotacticum strain MS-1, a strain related to AMB-1 that produces more equidimensional particles than MV-1 [Kobayashi, et al., 2006]. In the case of MS-1, ultrasonication does not produce many side-by-side crystal pairs. Instead, ultrasonication of MS-1 tends to cause chains to string together in a head-to-tail fashion. The difference between the collapse styles of MV-1 and MS-1 is likely attributable to the energetic differences between elongate and equidimensional particles.

Cells of wild-type AMB-1 produce magnetite particles with a mean particle length of $\sim 35$ $\mathrm{nm}$ and length-to-width ratio $\sim 1.2$ (Fig. 3a,d,f). In powder A1, derived from freeze-dried wild- 
type AMB-1, $\sim 65 \%$ by volume of the crystals we measured were in chains of at least 2 particles and $\sim 35 \%$ were isolated. Previous observations of whole cells of wild-type AMB-1 indicate that single cells often produce chains with segments of anywhere between 1 and 21 crystals separated by gaps. The presence of isolated crystals in the freeze-dried powder is likely due to a combination of gaps in chains produced by single cells and disaggregation during the freezedrying process.

Cells of the AMB-1 mutant mnm 13 produce elongated crystals, with a mean length of $\sim 25 \mathrm{~nm}$ and a mean length-to-width ratio of $\sim 1.5$ (Fig 3b,e,h). About $90 \%$ by volume of the crystals produced by $m n m 13$ are isolated and $\sim 10 \%$ are in chains of 2 or more particles. Among particles with a length $>25 \mathrm{~nm}$, which dominate by volume and control the magnetic properties, the mean length-to-width ratio is $\sim 1.75$. Some of the bias toward greater elongation in larger crystals is likely observational; an elongate particle, viewed down the axis of elongation, appears to have a width/length ratio of 1 and a shorter length than its true length.

Cells of the mutant mnm 18 produce more equidimensional crystals, similar to those produced by the wild-type, with a mean length of $\sim 40 \mathrm{~nm}$ and a mean length-to-width ratio of $\sim 1.2$ (Fig 3c,f,i). By volume, $\sim 65 \%$ of the particles are isolated and $\sim 35 \%$ are in chains. Though most of the chains consist of only two particles, they can grow significantly longer. The longest mnm 18 chain we observed consisted of seven particles, which suggests that a small fraction of mnm 18 cells exhibit the wild-type phenotype.

\section{Ferromagnetic resonance spectroscopy}

Our measurements of the FMR spectra of intact MV-1 and wild-type AMB-1 agree with those of [Weiss, et al., 2004], exhibiting distinctive asymmetric spectra that are extended in the low field direction. MV-1 has a broader spectrum than AMB-1, which reflects the greater anisotropy of its magnetite chains, generated by particle elongation as well as chain alignment (Table 1; Fig. 4; Fig. 5a). MV-1 also has three characteristic maxima in the derivative spectrum, seen in samples V1, V2a, and V2b at $\sim 180 \mathrm{mT}, \sim 300 \mathrm{mT}$, and $\sim 350 \mathrm{mT}$. Our one-component model spectra are unable to reproduce this trait. Our attempts to fit these three spectra with twocomponent models, however, yielded disparate secondary fit components with no clear physical interpretation. This disparity suggests the values thus determined were artifacts, and we therefore report the single-component fits in Table 2. A more complete physical model capable of including multiple sources of anisotropy might explain the distinctive triple maxima of MV-1 spectra.

Based on the demagnetization factors derived by [Osborn, 1945] and assuming that the anisotropy is dominated by uniaxial shape anisotropy, the $B_{a n}$ value of $171 \mathrm{mT}$ fitted to the spectrum of intact MV-1 (sample V1) is that expected from prolate spheroids of stoichiometric magnetite $\left(M_{s}=480 \mathrm{kA} / \mathrm{m}\right)$ with length-to-width ratios of $\sim 2.35$. The calculated ratio is significantly larger than that observed for individual particles under TEM and therefore likely reflects the joint contribution of particle elongation and chain structure. The large, positive $B_{a n}$ value indicates that the negative contribution of magnetocrystalline anisotropy is overwhelmed by the positive contributions of shape anisotropy and chain structure.

Ultrasonication of MV-1 broadens the FMR spectrum, with $\triangle B_{F W H M}$ increasing from 127 $\mathrm{mT}$ in sample V1 to 219 in sample V2a. This broadening, which is reflected in the spectral fits by an increase of $\sigma$ from $17 \mathrm{mT}$ to $27 \mathrm{mT}$ despite a slight decline in $B_{a n}$, suggests an increase in the heterogeneity of particle arrangement without the formation of strongly interacting clumps. 
Table 1. Measured Ferromagnetic Resonance Parameters

\begin{tabular}{|c|c|c|c|c|c|c|}
\hline Sample & Strain & Treatment & $g_{\text {eff }}$ & $A$ & $\begin{array}{c}\Delta B_{\mathrm{FWHM}} \\
(\mathrm{mT})\end{array}$ & $\boldsymbol{A}$ \\
\hline$\overline{\mathrm{A} 1}$ & AMB-1 wild-type & untreated & 2.01 & 0.76 & 87 & 0.21 \\
\hline A2 & AMB-1 wild-type & sonicated & 2.02 & 0.79 & 84 & 0.22 \\
\hline A3 & AMB-1 wild-type & SDS & 2.31 & 1.17 & 206 & 0.40 \\
\hline B1 & AMB-1 mnm 13 & untreated & 2.02 & 0.88 & 91 & 0.24 \\
\hline B2 & AMB-1 mnm 13 & sonicated & 2.01 & 0.86 & 95 & 0.24 \\
\hline B3 & AMB-1 mnm 13 & SDS & 2.02 & 0.83 & 107 & 0.25 \\
\hline $\mathrm{C} 1$ & AMB-1 mnm 18 & untreated & 2.07 & 1.13 & 80 & 0.27 \\
\hline $\mathrm{C} 2$ & AMB-1 mnm 18 & sonicated & 2.07 & 1.16 & 79 & 0.27 \\
\hline $\mathrm{C} 3$ & AMB-1 mnm 18 & SDS & 2.07 & 0.78 & 151 & 0.28 \\
\hline V1 & MV-1 & untreated & 1.78 & 0.35 & 127 & 0.18 \\
\hline V2a & MV-1 & sonicated & 1.84 & 0.29 & 219 & 0.26 \\
\hline $\mathrm{V} 2 \mathrm{~b}$ & MV-1 & $\begin{array}{l}\text { sonicated, } \\
4 \mathrm{~m} \text { dilution }\end{array}$ & 1.85 & 0.30 & 206 & 0.25 \\
\hline $\mathrm{V} 3 \mathrm{a}$ & MV-1 & SDS & 2.58 & 1.77 & 244 & 0.54 \\
\hline $\mathrm{V} 3 \mathrm{~b}$ & MV-1 & $\begin{array}{l}\text { SDS, } \\
1 \mathrm{~m} \text { dilution }\end{array}$ & 2.54 & 1.62 & 218 & 0.49 \\
\hline$V 3 c$ & MV-1 & $\begin{array}{l}\text { SDS, } \\
5 \mathrm{~m} \text { dilution }\end{array}$ & 1.86 & 0.25 & 208 & 0.25 \\
\hline
\end{tabular}

Although dilution (sample V2b) produces a significant increase in anhysteretic susceptibility (see discussion below), it results in little change in the FMR spectrum.

In contrast, lysis of MV-1 cells with SDS produces a drastic change in the FMR spectrum, as it causes the particles to collapse into clumps. The FMR spectra of these clumps, like the FMR spectra of similarly treated AMB-1 observed by [Weiss, et al., 2004] and [Kopp, et $a l ., 2006]$ and in the present work, are broad and exhibit high-field extended asymmetry reflective of a negative effective anisotropy field. The negative anisotropy may reflect the anisotropy of the surface of particle clumps or the oblateness of the clumps. Although modeling clumps with expressions derived for isolated particles is far from ideal, the fitted $B_{a n}$ value of $120 \mathrm{mT}$ corresponds to that predicted for oblate spheroids with a length-to-width ratio of $\sim 0.62$. [Griscom, et al., 1988] observed similar traits in the spectra of powders of magnetite nanoparticles exhibiting planar interactions.

Subsequent dilution causes the gradual reappearance of positive anisotropy, again as in the case of AMB-1 [Kopp, et al., 2006]. After one minute of dilution, the spectrum is best fit by a two-component model, with $84 \%$ of the absorption caused by a component with $B_{a n}$ of $-130 \mathrm{mT}$ and $16 \%$ caused by a component with $B_{a n}$ of $157 \mathrm{mT}$. The former component likely corresponds to particles in clumps, while the latter component likely corresponds to particles that are either isolated or in strings. After five minutes of dilution, the component with positive anisotropy dominates the spectrum (Fig. 5).

The spectrum of untreated cells of mnm 13 is not markedly different from that of wildtype AMB-1 (Fig. 5b). Although the empirical asymmetry parameter $A$ for $m n m 13$ reflects a lesser degree of asymmetry than the wild-type, this represents a failure of the empirical 
Table 2. Ferromagnetic Resonance Spectral Fits

\begin{tabular}{|c|c|c|c|c|c|c|c|c|c|c|c|c|c|}
\hline \multirow{2}{*}{$\begin{array}{l}\text { Sample } \\
\text { A1 }\end{array}$} & $\begin{array}{c}\text { Component } \\
\text { weight }\end{array}$ & \multicolumn{3}{|c|}{$g$} & \multicolumn{3}{|c|}{$B_{a n}(\mathrm{mT})$} & \multicolumn{3}{|c|}{$K_{2} / K_{1}$} & \multicolumn{3}{|c|}{$\sigma(\mathrm{mT})$} \\
\hline & $100 \%$ & 2.07 & \pm & 0.00 & 69.1 & \pm & 0.8 & -0.12 & \pm & 0.01 & 24.2 & \pm & 0.2 \\
\hline $\mathrm{A} 2$ & $100 \%$ & 2.07 & \pm & 0.00 & 63.6 & \pm & 0.7 & -0.13 & \pm & 0.01 & 23.5 & \pm & 0.2 \\
\hline \multirow[t]{2}{*}{ A3 } & $61 \%$ & 2.15 & \pm & 0.03 & 87.4 & \pm & 10.1 & & & & 55.5 & \pm & 2.4 \\
\hline & $39 \%$ & 2.38 & \pm & 0.01 & -171.2 & \pm & 4.3 & & & & 31.7 & \pm & 1.5 \\
\hline B1 & $100 \%$ & 2.08 & \pm & 0.00 & 90.9 & \pm & 2.5 & -0.32 & \pm & 0.02 & 31.1 & \pm & 0.3 \\
\hline B2 & $100 \%$ & 2.09 & \pm & 0.00 & 104.3 & \pm & 2.5 & -0.31 & \pm & 0.01 & 31.8 & \pm & 0.2 \\
\hline B3 & $100 \%$ & 2.10 & \pm & 0.00 & 99.7 & \pm & 1.5 & -0.23 & \pm & 0.01 & 34.2 & \pm & 0.2 \\
\hline \multirow[t]{2}{*}{$\mathrm{C} 1$} & $70 \%$ & 2.05 & \pm & 0.00 & -47.3 & \pm & 0.8 & & & & 19.2 & \pm & 0.1 \\
\hline & $30 \%$ & 2.12 & \pm & 0.01 & 76.1 & \pm & 1.1 & & & & 22.1 & \pm & 0.8 \\
\hline \multirow[t]{2}{*}{$\mathrm{C} 2$} & $57 \%$ & 2.06 & \pm & 0.00 & -43.0 & \pm & 0.6 & & & & 18.4 & \pm & 0.2 \\
\hline & $43 \%$ & 2.09 & \pm & 0.01 & 64.1 & \pm & 3.1 & & & & 28.2 & \pm & 0.6 \\
\hline \multirow[t]{2}{*}{$\mathrm{C} 3$} & $68 \%$ & 2.05 & \pm & 0.01 & 50.2 & \pm & 5.4 & & & & 43.1 & \pm & 0.8 \\
\hline & $32 \%$ & 2.34 & \pm & 0.01 & -142.3 & \pm & 3.3 & & & & 30.4 & \pm & 0.9 \\
\hline V1 & $100 \%$ & 2.21 & \pm & 0.01 & 170.9 & \pm & 2.6 & -0.03 & \pm & 0.01 & 17.3 & \pm & 0.3 \\
\hline V2a & $100 \%$ & 2.26 & \pm & 0.01 & 164.0 & \pm & 3.2 & 0.01 & \pm & 0.01 & 26.7 & \pm & 0.6 \\
\hline $\mathrm{V} 2 \mathrm{~b}$ & $100 \%$ & 2.24 & \pm & 0.01 & 160.4 & \pm & 2.7 & 0.00 & \pm & 0.01 & 24.9 & \pm & 0.4 \\
\hline V3a & $100 \%$ & 2.35 & \pm & 0.01 & -120.0 & \pm & 2.3 & 0.23 & \pm & 0.02 & 56.9 & \pm & 0.8 \\
\hline \multirow[t]{2}{*}{$\mathrm{V} 3 \mathrm{~b}$} & $84 \%$ & 2.37 & \pm & 0.01 & -129.9 & \pm & 2.6 & & & & 58.3 & \pm & 0.9 \\
\hline & $16 \%$ & 2.24 & \pm & 0.01 & 157.4 & \pm & 2.5 & & & & 21.2 & \pm & 0.7 \\
\hline V3c & $100 \%$ & 2.24 & \pm & 0.01 & 132.6 & \pm & 3.4 & 0.08 & \pm & 0.02 & 20.6 & \pm & 0.6 \\
\hline
\end{tabular}

The dominant components of $\mathrm{C} 1$ and $\mathrm{C} 2$ are modeled using cubic anisotropy. All other components are modeled using uniaxial anisotropy.

parameter; the fitted spectra reveal that $m n m 13$ in fact has a somewhat stronger anisotropy field than the wild-type, which reflects the particle elongation. The wild-type has a fitted $B_{a n}$ of 69 $\mathrm{mT}$, corresponding to a length-to-width ratio of $\sim 1.35$, while $m n m 13$ has a fitted $B_{a n}$ of $91 \mathrm{mT}$, corresponding to a length-to-width ratio of $\sim 1.50$. As with MV-1, the ratio calculated for the wild-type exceeds the value observed under TEM for individual particles, likely due to the effect of the chain structure in increasing $B_{a n}$. In contrast, the ratio calculated for $m n m 13$ corresponds to that observed under TEM.

Both sonication and lysis with SDS cause a slight increase in the fitted anisotropy field of $m n m 13$, which may reflect the formation of short strings of particles. In contrast, while sonication has only slight effect on the wild-type, treatment of the wild-type with SDS leads to a broader spectrum that is best fit by a two-component model in which $61 \%$ of particles have positive anisotropy $\left(B_{a n}=87 \mathrm{mT}\right)$ and $39 \%$ have negative anisotropy $\left(B_{a n}=-171 \mathrm{mT}\right)$. The latter component may reflect clumping. The absence of clumps in SDS-treated mnm 13 suggests that the greater diluteness of the particles prevents them from clumping.

The mutant $\mathrm{mnm} 18$ has an extremely distinctive spectrum (Fig. 5c). It is the only untreated magnetotactic bacterium measured so far that has $A>1$, which reflects the negative magnetocrystalline anisotropy of isolated particles of equidimensional magnetite. It provides the best example of a spectrum that can be fitted as a mixture, as it is the mixture of two components with clear physical interpretations corresponding to TEM observations. The intact $m n m 18$ is a mixture composed $70 \%$ of a component with negative anisotropy $\left(B_{a n}=-47 \mathrm{mT}\right)$ and $30 \%$ of a 
positive anisotropy component with parameters closely resembling those of the wild-type $\left(B_{a n}=\right.$ $76 \mathrm{mT}$ ). From the FMR data, we can predict that, by volume, the sample consists $70 \%$ of isolated crystals and $30 \%$ of chains of at least 2 crystals in length. These proportions are in close agreement with the values $(65 \%$ and $35 \%)$ estimated from the TEM images, which confirms the proposal by [Weiss, et al., 2004] that the uniquely asymmetric FMR spectrum of magnetotactic bacteria results primarily from the alignment of crystals in chains. We can also use this composition to unmix the isolated crystals from the other rock magnetic parameters, taking the properties of the wild-type to represent those of the fraction in chains.

The isolated component has a narrower Gaussian linewidth $(\sigma=20 \mathrm{mT})$ than is typical of most magnetotactic bacteria, which may reflect that lesser degree of heterogeneity possible with isolated crystals than with arrangements of crystals. The anisotropy measured for isolated crystals of $m n m 18$ is slightly less than that expected for isolated crystals of stoichiometric magnetite dominated by cubic anisotropy, which would have $B_{a n}$ of about $-56 \mathrm{mT}$. The reduced anisotropy constant $\left(K_{1} \approx 1.1 \times 10^{4} \mathrm{~J} / \mathrm{m}^{3}\right)$ may result from minor non-stochiometry $(\sim 0.4 \%$ cation depletion) [Kakol and Honig, 1989], which is consistent with the reduced Verwey transition temperature of $\sim 100 \mathrm{~K}$ observed in AMB-1 magnetite (Fig. 6e) [Muxworthy and McClellan, 2000].

Sonication of $m n m 18$ cells leads to a slight increase in the proportion in chains, while treatment with SDS drastically alters the spectrum. SDS-treated cells of mnm 18 come to resemble those of the wild-type more closely, because the sample becomes dominated by short linear strings of particles, the anisotropy of which is controlled primarily by particle arrangement. The fitted spectrum consists $68 \%$ of a component with positive anisotropy $\left(B_{a n}=\right.$ $50 \mathrm{mT})$ and $32 \%$ of a component with strong negative anisotropy $\left(B_{a n}=-142 \mathrm{mT}\right)$ comparable to those of clumps formed in SDS treatment of wild-type AMB-1 and MV-1. Thus, the comparison of the unmixed components of intact and SDS-treated mnm 18 provides powerful insight into the role of chain formation in controlling the magnetic properties of magnetotactic bacteria.

\section{Isothermal remanent magnetization}

The room-temperature IRM acquisition coercivity spectra for cells of wild-type AMB-1 and MV-1, regardless of treatment, agree in general shape, though not in precise parameterization, with the biogenic soft and biogenic hard components recognized by Egli [Egli, 2004] (Fig. 6a; Table 3). MV-1 has a narrow peak centered at a median field of $55 \mathrm{mT}$, while AMB-1 has a broader peak centered at $27 \mathrm{mT}$. The mutant $m n m 13$ is slightly softer than the wild-type (median field of $23 \mathrm{mT}$ ), which may be due to the smaller volume of $\mathrm{mnm} 13$ particles. The mutant mnm 18 is both softer and has a broader spectrum than the other strains (median field of $16 \mathrm{mT}$ ). When FMR analyses and TEM observations are used to guide the unmixing of the chains and solitary particles in $m n m 18$, the solitary particles are revealed to have a spectrum with a median coercive field of $11 \mathrm{mT}$. The drastic difference between the isolated, equidimensional particles produced by $m n m 18$ and the elongate particles of $m n m 13$, as well as the chains of equidimensional particles in the wild-type cells, highlights the role of these traits in stabilizing the magnetic moments of magnetotactic bacteria.

For all samples of unlysed cells of AMB-1, both wild-type and mutant, acquisition and demagnetization curves align fairly closely (Figs. 6a and 6b); Cisowski $R$ values are all $\geq 0.42$, and the median destructive field falls within $5 \mathrm{mT}$ of the median acquisition field. This is not the case for the SDS-treated mutants and both the SDS-treated and the ultrasonicated wild-types, 
Table 3. Room Temperature Rock Magnetic Parameters

\begin{tabular}{lrrrrrrr} 
Sample & $\begin{array}{c}\boldsymbol{H}_{\boldsymbol{c r}} \\
(\mathbf{m T})\end{array}$ & $\boldsymbol{R}$ & $\begin{array}{c}\text { MAF of } \\
\text { IRM } \\
(\mathbf{m T})\end{array}$ & $\begin{array}{c}\text { MDF of } \\
\text { IRM } \\
(\mathbf{m T})\end{array}$ & $\begin{array}{c}\text { MDF of } \\
\mathbf{A R M} \\
(\mathbf{m T})\end{array}$ & $\begin{array}{c}\boldsymbol{k}_{\text {ARM }} / \mathbf{I R M} \\
(\mathbf{m m} / \mathbf{A})\end{array}$ & $\begin{array}{r}\text { Predicted } \\
\text { switching } \\
\text { field (mT) }\end{array}$ \\
\hline A1 & 24.0 & 0.44 & 26.5 & 21.6 & 22.2 & 2.93 & 30.3 \\
A2 & 22.0 & 0.43 & 24.0 & 19.8 & 21.1 & 2.07 & 27.8 \\
A3 & 16.6 & 0.29 & 24.1 & 10.2 & 17.4 & 0.64 & 59.8 \\
B1 & 21.3 & 0.42 & 23.4 & 19.3 & 19.3 & 1.37 & 31.0 \\
B2 & 23.9 & 0.42 & 26.6 & 22.2 & 22.3 & 1.26 & 35.7 \\
B3 & 26.0 & 0.39 & 30.7 & 21.8 & 24.2 & 1.29 & 38.2 \\
C1 & 14.5 & 0.47 & 15.7 & 13.8 & 13.7 & 3.55 & 26.7 \\
C1' & 10.7 & 0.44 & 10.6 & 9.9 & 10.5 & 3.99 & 22.3 \\
C2 & 14.7 & 0.44 & 16.6 & 13.8 & 13.8 & 3.25 & 24.4 \\
C3 & 21.7 & 0.35 & 26.7 & 16.6 & 21.9 & 1.38 & 39.8 \\
V1 & 57.8 & 0.42 & 55.3 & 61.2 & 65.8 & 1.79 & 82.9 \\
V2a & 55.1 & 0.27 & 63.3 & 45.9 & 58.1 & 0.41 & 82.8 \\
V2b & 48.4 & 0.31 & 55.0 & 41.7 & 49.8 & 1.59 & 80.2 \\
V3a & 28.4 & 0.14 & 43.2 & 17.0 & 24.7 & 0.10 & 73.8 \\
V3b & n.d. & n.d. & n.d. & n.d. & 52.7 & 0.66 & 67.2 \\
V3c & 52.3 & 0.34 & 52.5 & 46.5 & 56.3 & 1.68 & 71.6 \\
\hline
\end{tabular}

$\mathrm{C} 1$ ' is the unmixed endmember of $\mathrm{C} 1$ composed of isolated particles. Step-wise IRM curves were not measured for V3b. Predicted switching field is calculated from the FMR fit parameters as described in the text.

which reflects greater interparticle magnetostatic interactions in the wild-types than in the mutants. Notably, the IRM acquisition curve of SDS-treated mnm 18 closely resembles that of the wild-type (median field of $27 \mathrm{mT}$ ), while the demagnetization curve remains closer to that of the untreated mnm 18. As FMR data indicate the formation of linear strings of particle in the SDStreated $m n m 18$, the observation may suggest that IRM acquisition coercivity is more strongly affected by chain structures than is demagnetization coercivity.

\section{Anhysteretic remanent magnetization}

The ARM acquisition curves for wild-type AMB-1 and MV-1 are consistent with previous measurements [Moskowitz, et al., 1993; Moskowitz, et al., 1988] (Fig. 6c, 6d; Table 3). MV-1 has markedly lower anhysteretic susceptibility than AMB-1. Two factors likely contribute to this difference. First, as seen in the TEM images, untreated MV-1 has undergone a greater degree of chain collapse than untreated AMB-1, due to the intrinsic instability of chains of elongate particles. The increased three-dimensional magnetostatic interactions in collapsed chains serve to lower ARM susceptibility. Second, elongate particles have a higher switching field and thus lower intrinsic ARM susceptibility than more equidimensional particles of the same volume (see Fig. 11 of [Egli, 2003]).

The pattern of variation of ARM susceptibility of lysed MV-1 shows some notable differences from parallel experiments previously reported for AMB-1 [Kopp, et al., 2006]. For 
both strains, ultrasonicated bacteria exhibit a lower susceptibility than untreated bacteria and a higher susceptibility than SDS-treated bacteria. But whereas dilution of ultrasonicated AMB-1 produced little change in ARM susceptibility, dilution of ultrasonicated MV-1 produces significant change. Undiluted ultrasonicated MV-1 exhibits a similar susceptibility to SDStreated MV-1 diluted for 1 minute, and ultrasonicated MV-1 diluted for 4 minutes exhibits a similar susceptibility to SDS-treated MV-1 diluted for five minutes. The difference between the strains again likely reflects differences in collapse style between equidimensional particles and elongate particles; the strings produced by ultrasonication of AMB-1 are less likely to be reconfigured during dilution than the meshes produced by ultrasonication of MV-1.

The crystals produced by mnm 13 have even lower anhysteretic susceptibility than MV-1, a reflection of the combined influence of their elongation and their smaller size. In fact, their ARM susceptibility lies significantly above what would be predicted based on TEM measurements. [Egli and Lowrie, 2002] calculate that a particle with a length-to-width ratio of 1.9 and a cube root of volume of $\sim 20 \mathrm{~nm}$ should have a $k_{A R M} / \mathrm{IRM}$ ratio of about $0.5 \mathrm{~mm} / \mathrm{A}$, whereas the measured value is $1.4 \mathrm{~mm} / \mathrm{A}$. Given the measured median destructive field, the ARM susceptibility measured would be expected for particles with a length of $45 \mathrm{~nm}$ and a length-to-width ratio of 1.3 .

The isolated particles in untreated cells of mnm 18 produce one of the highest ARM susceptibilities that we have ever observed. With a $k_{A R M} / I R M$ of $4.0 \mathrm{~mm} / \mathrm{A}$, they lie among the highest sediment values tabulated by [Egli, 2004], and above previously measured magnetotactic bacteria [Moskowitz, et al., 1993]. Given the similarity of the crystals produced by $m n m 18$ to those produced by the wild-type, the high $k_{A R M} / I R M$ is likely due to the absence of magnetostatic interactions. Although they have less effect than three-dimensional interactions, even the linear interactions in wild-type AMB-1 appear to lower lower ARM susceptibility slightly.

At biasing fields below $300 \mu \mathrm{T}$, the ARM/IRM curves of ultrasonicated mnm13 (B2), SDS-treated mnm 13 (B3), and SDS-treated mnm 18 (C3) are almost identical, whereas above 300 $\mu \mathrm{T}$ they diverge, with $\mathrm{B} 2>\mathrm{B} 3>\mathrm{C} 3$. The divergence may reflect the presence of a greater proportion of more strongly interacting particles (which acquire ARM in higher biasing fields) in the more severely treated samples.

\section{Low-temperature magnetic properties}

Regardless of treatment, the MV-1 samples have low $\delta_{\mathrm{FC}} / \delta_{\mathrm{ZFC}}$ : the untreated and ultrasonicated samples have $\delta_{\mathrm{FC}} / \delta_{\mathrm{ZFC}}$ of 1.4 , while the SDS-treated MV-1 has $\delta_{\mathrm{FC}} / \delta_{\mathrm{ZFC}}$ of 1.1 (Fig. 6e; Table 4). Based on the criterion of [Moskowitz, et al., 1993], $\delta_{\mathrm{FC}} / \delta_{\mathrm{ZFC}}>2$ indicates the presence of chains. The reason why our untreated MV-1 fails this test is unclear, although such low values have previously been observed for some fresh cultures of MV-1 (B. Moskowitz, pers. comm.). The low values may be related to the partial chain collapse previously described, but they stand in contrast to FMR data indicating the presence of chains. They are not a product of accidental sample oxidation; the absolute values of $\delta_{\mathrm{FC}}$ and $\delta_{\mathrm{ZFC}}$ are relatively large.

The untreated cells of mutant $m n m 13$ fail the Moskowitz test, with $\delta_{\mathrm{FC}} / \delta_{\mathrm{ZFC}}=1.9$, consistent with the absence of chains in this sample. Inspection of its low-temperature demagnetization curves indicates that the sample's low-temperature properties are dominated by the unblocking of superparamagnetic grains, in agreement with the smaller grain size observed in the TEM images. In contrast, the untreated cells of $m n m 18$ have $\delta_{\mathrm{FC}} / \delta_{\mathrm{ZFC}}=2.6$, which slightly exceeds the wild-type value of 2.5 even though less than half of the crystals present are in chains. 
Table 4. Low-Temperature Magnetic Parameters

\begin{tabular}{lcrc} 
Sample & $\boldsymbol{\delta}_{\boldsymbol{Z} \boldsymbol{F C}}$ & $\boldsymbol{\delta}_{\boldsymbol{F} \boldsymbol{C}} \boldsymbol{\delta}_{\boldsymbol{Z} \boldsymbol{F C}}$ & \multicolumn{1}{c}{$\boldsymbol{f}_{\boldsymbol{L} \boldsymbol{T C}}$} \\
\hline A1 & 0.13 & 2.53 & 0.98 \\
B1 & 0.17 & 1.07 & 0.97 \\
C1 & 0.16 & 2.57 & 0.83 \\
C3 & 0.35 & 1.24 & 0.86 \\
V1 & 0.29 & 1.40 & 0.90 \\
V2a & 0.29 & 1.42 & 0.94 \\
V3a & 0.61 & 1.10 & 0.77 \\
\hline
\end{tabular}

The unexpected result cannot be explained by nonstoichiometry, which would increase $\delta_{\mathrm{FC}} / \delta_{\mathrm{ZFC}}$ while at the same time decreasing $\delta_{\mathrm{FC}}$ and $\delta_{\mathrm{ZFC}}$ [Carter-Stiglitz, et al., 2004]. No such drop in $\delta_{\mathrm{FC}}$ and $\delta_{\mathrm{ZFC}}$ is observed. Furthermore, whereas SDS-treatment of mnm 18 produces an FMR spectrum reflecting the presence of linear particle arrangements, it also causes $\delta_{\mathrm{FC}} / \delta_{\mathrm{ZFC}}$ to drop to 1.2, comparable to the SDS-treated wild-type [Kopp, et al., 2006]. The elevated $\delta_{\mathrm{FC}} / \delta_{\mathrm{ZFC}}$ ratios of mnm 18 may occur because the chain component within the sample has a higher $\delta_{\mathrm{FC}} / \delta_{\mathrm{ZFC}}$ than the wild-type AMB-1 that we measured; previously observed $\delta_{\mathrm{FC}} / \delta_{\mathrm{ZFC}}$ ratios for AMB-1 range as high as 5.9 [Weiss, et al.,

2004]. Alternatively, the distinctive $\delta_{\mathrm{FC}} / \delta_{\mathrm{ZFC}}$ ratios of magnetotactic bacteria may be due, at least in part, to same factor other than chain structure and non-stoichiometry.

Consistent with prior measurements of wild-type AMB-1 [Kopp, et al., 2006], SDStreated $m n m 18$ exhibits an increase in remanence on cooling through the Verwey temperature, while intact $m n m 18$ exhibits a decrease in remanence. In contrast, both intact and SDS-treated MV-1, like intact AMB-1, exhibit a decrease in remanence upon cooling through the Verwey transition (Fig. 6f). We have no explanation for this phenomenon.

\section{Discussion}

As measures of magnetic anisotropy and magnetostatic interaction, FMR parameters should be related to other magnetic properties that are a function of these characteristics. In so far as it possible to fit spectra well and thus obtain an accurate measurement of the anisotropy field of a sample, it is possible to use FMR spectra to estimate the switching field distribution of a sample. Neglecting thermal energy, the median coercive field of a sample is given by $B_{c} \approx \frac{1}{2}\left|B_{a n}\left(1+K_{2} / K_{1}\right)\right|\left[\right.$ Dunlop and $\ddot{O}_{z}$ demir, 1997]. A plot of the calculated $B_{c}$ against the median acquisition field of IRM acquisition is shown in Figure $7 \mathrm{a}$.

There is a good correlation between the two parameters, although the estimates derived from the FMR spectra are significantly higher than the measured values. The discrepancy is largely accounted for by the thermal fluctuation field, which for $100 \mathrm{~nm}$ cubes of magnetite at room temperature is approximately $50 \sqrt{B_{c}}$, or about $10 \mathrm{mT}$ for particles with $B_{c}=30 \mathrm{mT}$ [Dunlop and Özdemir, 1997]. Linear regression of the $B_{c}$ values for mnml3 and mnm18, with the slope of the line fixed at 1 because of the expected theoretical relationship between $B_{c}$ and MAF, yields the line $B_{c}=\mathrm{MAF}+9.7 \mathrm{mT}$, with a coefficient of determination $r^{2}=0.89$. Removing the constraint on the slope does not significantly improve the fit. The y-intercept thus calculated is in agreement with the expected thermal fluctuation field.

Cells of mutant AMB-1 and intact cells of wild-type AMB-1 have $B_{c}$ close to those predicted from the regression line, but SDS-treated cells of AMB-1 and all MV-1 samples fall well off the line. This difference may be due to a combination of imperfect fitting of the FMR spectra and the presence of additional factors not treated in the simple physical model used to predict $B_{c}$.

There is no single parameter that perfectly reflects interaction field strength [Dunlop, et $a l ., 1990]$, but the crossover $R$ value of Cisowski [Cisowski, 1981] is commonly used. The 
strength of three-dimensional magnetostatic interactions affects two parameters employed in modeling FMR spectra: the anisotropy field $B_{a n}$ and the Gaussian linewidth $\sigma$. Local anisotropy in magnetostatic interactions, such as that which occurs on the surface of a clump of particles, alters the anisotropy field, while the heterogeneity of local magnetic environments produced by interactions results in an increase in Gaussian linewidth. Other factors also contribute to both these terms, however, so neither provides a good measure of interaction field strength. The empirical linewidth parameter $\Delta B_{\mathrm{FWHM}}$ appears to provide a better measure, as it correlates reasonably well with the Cisowski $R$ parameter (Fig. 7b). Linear regression yields the relationship $\Delta B_{\mathrm{FWHM}}=373 \mathrm{mT}-632 \mathrm{mT} \times R$, with a coefficient of determination $r^{2}=0.84$. When present, strong three-dimensional interactions overwhelm other factors controlling $\Delta B_{\mathrm{FWHM}}$, such as single-particle anisotropy and linear interactions.

The bacterial samples measured in this work continue to support the use of the empirical discriminant factor $\alpha$ [Kopp, et al., 2006] to distinguish biogenic magnetite chains (Fig. 8). Of all the intact cells of magnetotactic bacteria we measured, only those of the mutant mnm 18 have $\alpha>$ 0.24 . This exception arises because $m n m 18$ has $A>1$ and, while $\alpha$ serves as a proxy for Gaussian linewidth $\sigma$ when $\sigma$ is around $30 \mathrm{mT}$ and $A<1$, it does not when $A>1$, as can be seen from the $\alpha$ contours plotted on Fig. 8. As can be seen from the contours on Fig. 8, mnm 18 falls within the domain of intact magnetotactic bacterial cells when $\sigma$ values of synthetic spectra are used to delineate boundaries.

Ultrasonication in general results in a slight increase in $\alpha$, which confirms prior results [Kopp, et al., 2006]. SDS treatments of the wild-type cells of both MV-1 and AMB-1 result in drastic shifts in $\alpha$ as highly interacting clumps come to dominate the sample. The increase in $\alpha$ that occurs with SDS-treatment of cells of the AMB-1 mutants, in which the magnetite is more dilute, is present but subtle. SDS-treated cells of both wild-type strains, when diluted by mixing for five minutes, experience a significant reduction in $\alpha$ to values characteristic of the domain previously identified as being the magnetofossil domain, namely $\alpha<0.30$ [Kopp, et al., 2006].

In agreement with [Kopp, et al., 2006; Weiss, et al., 2004], these data support the use of ferromagnetic resonance spectroscopy as a technique for identifying potential magnetofossils in the sedimentary record. Because it can provide a rapid way of estimating the biogenic contribution to sedimentary magnetism, FMR has the potential to be a highly useful tool for environmental magnetism and magnetic paleobiology.

\section{Conclusion}

We have generated mutant strains of magnetotactic bacteria that allow us to start to untangle the contributions of chain arrangement and particle elongation to the ferromagnetic resonance and rock magnetic properties of magnetotactic bacteria. The four strains we have analyzed represent all four possible combinations of chain and solitary particles, and elongate and equidimensional particles. In addition, the SDS-treated cells of mnm 18 allow us to investigate the changes that occur as solitary equidimensional particles assemble into linear structures. Our findings indicate that ferromagnetic resonance spectroscopy provides an effective technique for estimating the switching field distribution and interaction effects within a sample and continue to support the use of ferromagnetic resonance spectroscopy as a way of identifying magnetotactic bacteria and magnetofossils. Since it takes only a few minutes to acquire a FMR spectrum, which is significantly faster than most rock magnetic techniques being used for similar 
purposes, we hope that our work will spur the broader adoption of ferromagnetic resonance spectroscopy by the rock magnetic community.

\section{Selected Notation}

\begin{tabular}{|c|c|}
\hline \multicolumn{2}{|r|}{ FMR: Empirical Parameters } \\
\hline$A$ & Asymmetry ratio $=\Delta B_{\text {high }} / \Delta B_{\text {low }}$ \\
\hline$B_{\text {eff }}$ & Applied field at peak of integrated absorption spectrum, mT \\
\hline$\triangle B_{F W H M}$ & Full-width at half-maximum, $\Delta B_{\text {high }}+\Delta B_{\text {low }}$ \\
\hline$\Delta B_{\text {high }}\left(\Delta B_{\text {low }}\right)$ & $\begin{array}{l}\text { Half-width at half-maximum of integrated spectrum on high-field (low-field) side of } \\
\text { peak, mT }\end{array}$ \\
\hline$g_{\text {eff }}$ & $g$ value at absorption peak, $h v / \beta B_{\text {eff }}$ \\
\hline$\alpha$ & Empirical discriminant factor, $0.17 \mathrm{~A}+9.8 \times 10^{-4} \mathrm{mT}^{-1} \cdot \Delta B_{F W H M}$ \\
\hline \multicolumn{2}{|r|}{ FMR: Physical Parameters } \\
\hline$B_{a n}$ & $\begin{array}{l}\text { Effective anisotropy field: } 2 K_{1} / M \text { for magnetocrystalline anisotropy, } \mu_{0} M_{s} \Delta N \text { for } \\
\text { shape anisotropy }\end{array}$ \\
\hline$g$ & True spectroscopic $g$-factor (equivalent to $g_{\text {eff }}$ when $B_{a n}=0$ ) \\
\hline$K_{2} / K_{1}$ & Ratio of second-order and first-order anisotropy constants \\
\hline$\sigma$ & Standard deviation of Gaussian broadening function \\
\hline \multicolumn{2}{|r|}{ Rock magnetic parameters } \\
\hline$H_{c r}$ & $\begin{array}{l}\text { Coercivity of remanence, determined here from intersection point of IRM } \\
\text { acquisition and demagnetization curves, } \mathrm{mT}\end{array}$ \\
\hline$f_{L T C}$ & $\begin{array}{l}\text { Fraction of room-temperature SIRM retained after cycling to low temperature and } \\
\text { back }\end{array}$ \\
\hline$k_{A R M} / \mathrm{IRM}$ & $\begin{array}{l}\text { ARM susceptibility normalized to IRM (measured here with } 0.1 \mathrm{mT} \text { ARM biasing } \\
\text { field, } 100 \mathrm{mT} \text { ARM alternating field, and } 100 \mathrm{mT} \text { IRM pulse field), } \mathrm{mm} / \mathrm{A}\end{array}$ \\
\hline$M A F(M D F)$ & $\begin{array}{l}\text { Median acquisition (destructive) fields, at which half of a total remanence is } \\
\text { acquired (destroyed), mT }\end{array}$ \\
\hline$R$ & $\begin{array}{l}\text { Cisowski R parameter, reflecting magnetostatic interactions: fraction of IRM } \\
\text { remaining at } H_{c r}\end{array}$ \\
\hline$\delta_{F C}\left(\delta_{Z F C}\right)$ & $\begin{array}{l}\left(J_{80 \mathrm{~K}}-J_{150 \mathrm{~K}}\right) / J_{80 \mathrm{~K}} \text { for field-cooled (zero-field-cooled) low-temperature SIRM } \\
\text { thermal demagnetization curves }\end{array}$ \\
\hline
\end{tabular}

\section{Acknowledgements}

We thank Angelo Di Bilio for assistance with the EPR spectrometer, Arash Komeili for assistance with the mutagenesis, and Mike Jackson, David Griscom, and an anonymous reviewer for helpful comments. The Beckman Institute provided support for the use of the MPMS. REK, JLK, and CZN would like to thank the Agouron Institute, the Moore Foundation, and the NASA Astrobiology Science and Technology Instrument Development program for support. AK was partially supported by funds from a New Energy and Industrial Technology Development Organization fellowship. DAB was supported by US National Science Foundation grant EAR-0311950. BPW thanks the NASA Mars Fundamental Research and NSF Geophysics Programs.

\section{References}

Carter-Stiglitz, B., et al. (2004), More on the low-temperature magnetism of stable single domain magnetite: Reversibility and non-stoichiometry, Geophys. Res. Lett., 31, L06606.

Chang, S. B. R., and J. L. Kirschvink (1989), Magnetofossils, the magnetization of sediments, and the evolution of magnetite biomineralization, Annu. Rev. Earth Planet. Sci., 17, 169-195.

Chang, S. B. R., et al. (1989), Biogenic magnetite in stromatolites. 2. Occurrence In ancient sedimentary environments, Precamb. Res., 43, 305-315. 
Cisowski, S. (1981), Interacting vs. non-interacting single-domain behavior in natural and synthetic samples, Phys. Earth Planet. Inter., 26, 56-62.

Dean, A. J., and D. A. Bazylinski (1999), Genome analysis of several marine, magnetotactic bacterial strains by pulsed-field gel electrophoresis, Curr. Microbiol., 39, 219-225.

Devouard, B., et al. (1998), Magnetite from magnetotactic bacteria: Size distributions and twinning, Am. Mineral., $83,1387-1398$.

Dunlop, D. J., and Ö. Özdemir (1997), Rock Magnetism: Fundamentals and Frontiers, 573 pp., Cambridge University Press, Cambridge.

Dunlop, D. J., et al. (1990), Preisach Diagrams and Anhysteresis - Do They Measure Interactions, Phys. Earth Planet. Inter., 65, 62-77.

Egli, R. (2003), Analysis of the field dependence of remanent magnetization curves, J. Geophys. Res., 108.

Egli, R. (2004), Characterization of individual rock magnetic components by analysis of remanence curves, 1. Unmixing natural sediments, Studia Geophys. Geodaet., 48, 391-446.

Egli, R., and W. Lowrie (2002), Anhysteretic remanent magnetization of fine magnetic particles, J. Geophys. Res., 107.

Griscom, D. L. (1974), Ferromagnetic resonance spectra of lunar fines: some implications of line shape analysis, Geochim. Cosmochim. Acta, 38, 1509-1519.

Griscom, D. L. (1981), Ferromagnetic resonance condition and powder pattern analysis for dilute, spherical, singledomain particles of cubic crystal structure, J. Magn. Reson., 45, 81-87.

Griscom, D. L., et al. (1988), Ferromagnetic-Resonance Studies of Iron-Implanted Silica, Nucl. Instrum. Methods Phys. Res., Sect. B, 32, 272-278.

Hanzlik, M., et al. (2002), Pulsed-field-remanence measurements on individual magnetotactic bacteria, J. Magn. Magn. Mater., 248, 258-267.

Kąkol, Z., and J. M. Honig (1989), Influence of deviations from ideal stoichiometry on the anisotropy parameters of magnetite $\mathrm{Fe}_{3(1-\delta)} \mathrm{O}_{4}$, Phys. Rev. B, 40, 9090-9097.

Kirschvink, J. L., and S. B. R. Chang (1984), Ultrafine-Grained Magnetite in Deep-Sea Sediments - Possible Bacterial Magnetofossils, Geology, 12, 559-562.

Kirschvink, J. L., and H. A. Lowenstam (1979), Mineralization and magnetization of chiton teeth: paleomagnetic, sedimentologic, and biologic implications of organic magnetite, Earth Planet. Sci. Lett., 44, 193-204.

Kittel, C. (1948), On the theory of ferromagnetic resonance absorption, Phys. Rev., 73, 155-161.

Kobayashi, A., et al. (2006), Experimental observation of magnetosome chain collapse in magnetotactic bacteria: sedimentological, paleomagnetic, and evolutionary implications, Earth Planet. Sci. Lett., 245, 538-550.

Komeili, A., et al. (2006), Magnetosomes are cell membrane invaginations organized by the actin-like protein MamK, Science, 311, 242-245.

Komeili, A., et al. (2004), Magnetosome vesicles are present before magnetite formation, and MamA is required for their activation, Proc. Natl. Acad. Sci., 101, 3839-3844.

Kopp, R. E., et al. (2006), Chains, clumps, and strings: Magnetofossil taphonomy with ferromagnetic resonance spectroscopy, Earth Planet. Sci. Lett., 10-25.

Moskowitz, B. M., et al. (1993), Rock magnetic criteria for the detection of biogenic magnetite, Earth Planet. Sci. Lett., 120, 283-300.

Moskowitz, B. M., et al. (1988), Magnetic Properties of Magnetotactic Bacteria, J. Magn. Magn. Mater., 73, 273288.

Muxworthy, A. R., and E. McClellan (2000), Review of the low-temperature magnetic properties of magnetite from a rock magnetic perspective, Geophys. J. Int., 140, 101-114.

Osborn, J. A. (1945), Demagnetizing Factors of the General Ellipsoid, Phys. Rev., 67, 351-357.

Pan, Y. X., et al. (2005), Rock magnetic properties of uncultured magnetotactic bacteria, Earth Planet. Sci. Lett., 237, 311-325.

Penninga, I., et al. (1995), Remanence Measurements on Individual Magnetotactic Bacteria Using a Pulsed Magnetic-Field, J. Magn. Magn. Mater., 149, 279-286.

Scheffel, A., et al. (2006), An acidic protein aligns magnetosomes along a filamentous structure in magnetotactic bacteria, Nature, 440, 110-114.

Schlömann, E. (1958), Ferromagnetic resonance in polycrystal ferrites with large anisotropy: General theory and application to cubic materials with a negative anisotropy constant, J. Phys. Chem. Solids, 6, 257-266.

Smit, J., and H. G. Beljers (1955), Ferromagnetic Resonance Absorption in $\mathrm{BaFe}_{12} \mathrm{O}_{19}$, a Highly Anisotropic Crystal, Philips Res. Rep., 10, 113-130. 
Thomas-Keprta, K. L., et al. (2000), Elongated prismatic magnetite crystals in ALH84001 carbonate globules: Potential Martian magnetofossils, Geochim. Cosmochim. Acta, 64, 4049-4081.

Weiss, B. P., et al. (2004), Ferromagnetic resonance and low temperature magnetic tests for biogenic magnetite, Earth Planet. Sci. Lett., 224, 73-89. 


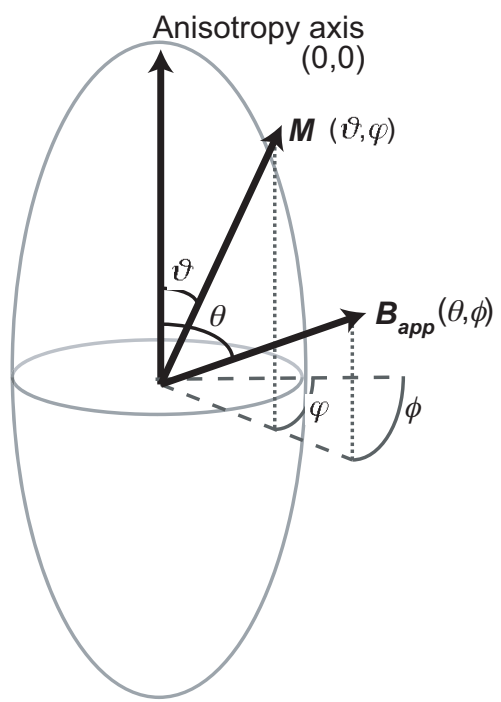

Figure 1. Angles used in the derivation of the resonance conditions. The origin of the reference frame is defined with respect to the anisotropy axis. The applied field $\boldsymbol{B}_{\text {app }}$ is oriented at azimuthal angle $\theta$ and declination. The magnetization $\boldsymbol{M}$ is oriented at azimuthal angle and declination . 

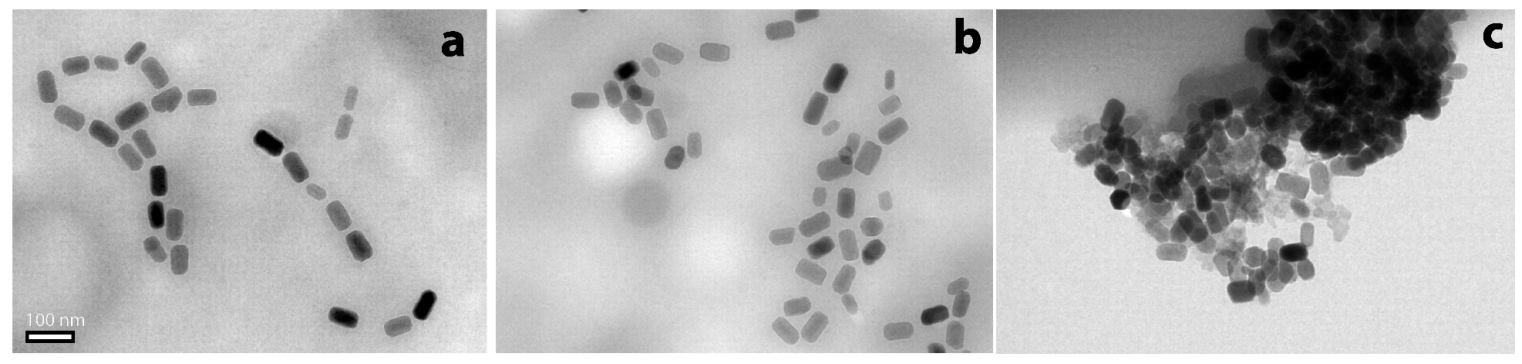

Figure 2. Transmission electron micrographs of MV-1. (a) Sample V1, untreated, (b) Sample V2a, ultrasonicated, and (c) Sample V3a, lysed with SDS. Scale bar is $100 \mathrm{~nm}$. 

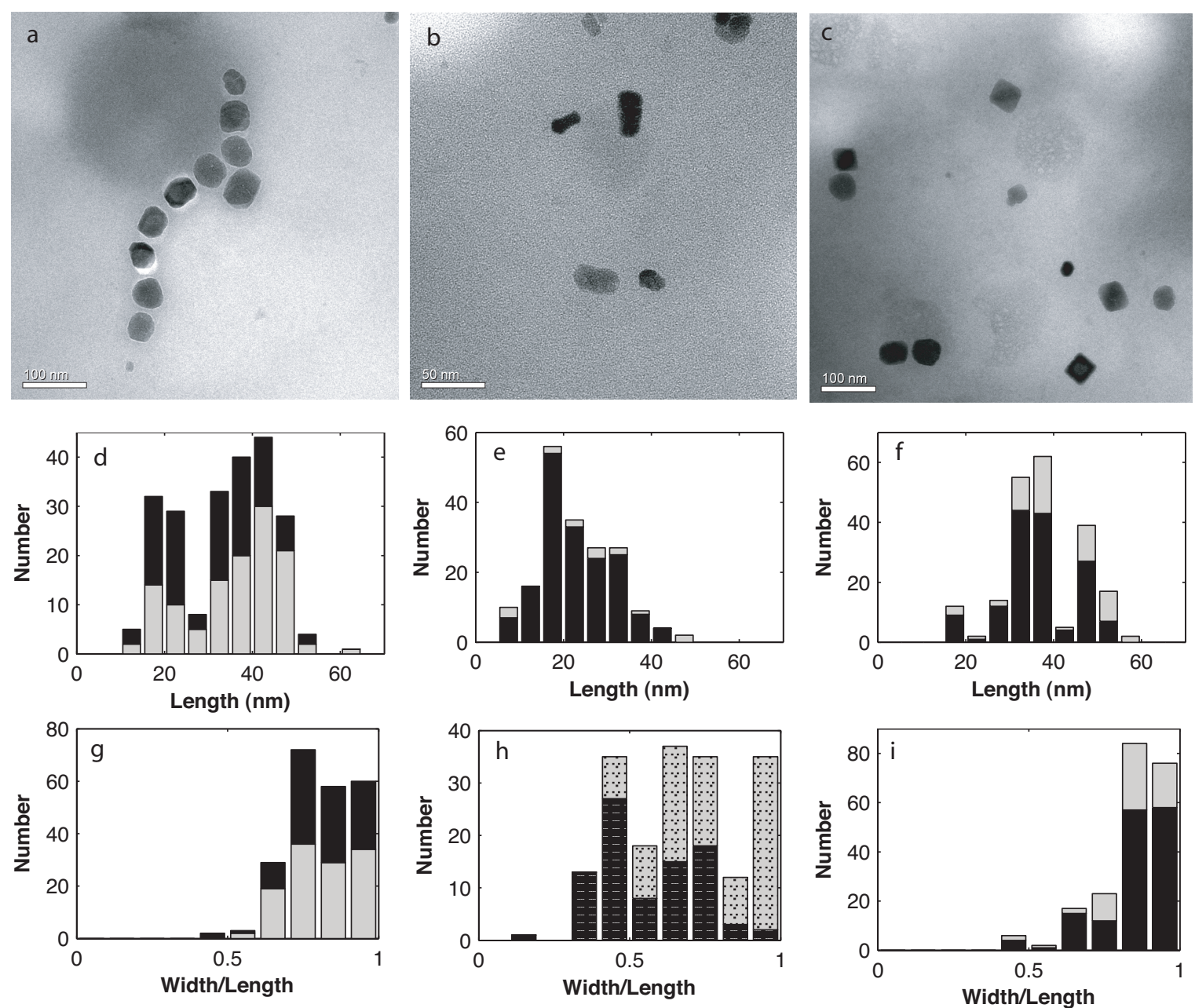

Figure 3. Transmission electron micrographs of and summary statistics for AMB-1 strains. (a-c) TEM images of freeze-dried powders of (a) wild-type, (b) mnm13, and (c) mnm18. In (a) and (c), scale bar is $100 \mathrm{~nm}$; in (b), scale bar is $50 \mathrm{~nm}$. (d-f) Histograms of particle length for magnetite produced by (d) wild-type, (e) mnm13, and (f) mnm 18. (g-i) Histograms of particle width/length ratios for magnetite produced by (g) wild-type, (h) mnm 13, and (i) mnm 18. In (d-g) and (i), dark bars represent particles in chains and light bars represent isolated particles. In (h), dark bars represent particles with length $\geqq 25 \mathrm{~nm}$ and light bars represent particles with length $<25 \mathrm{~nm}$. 


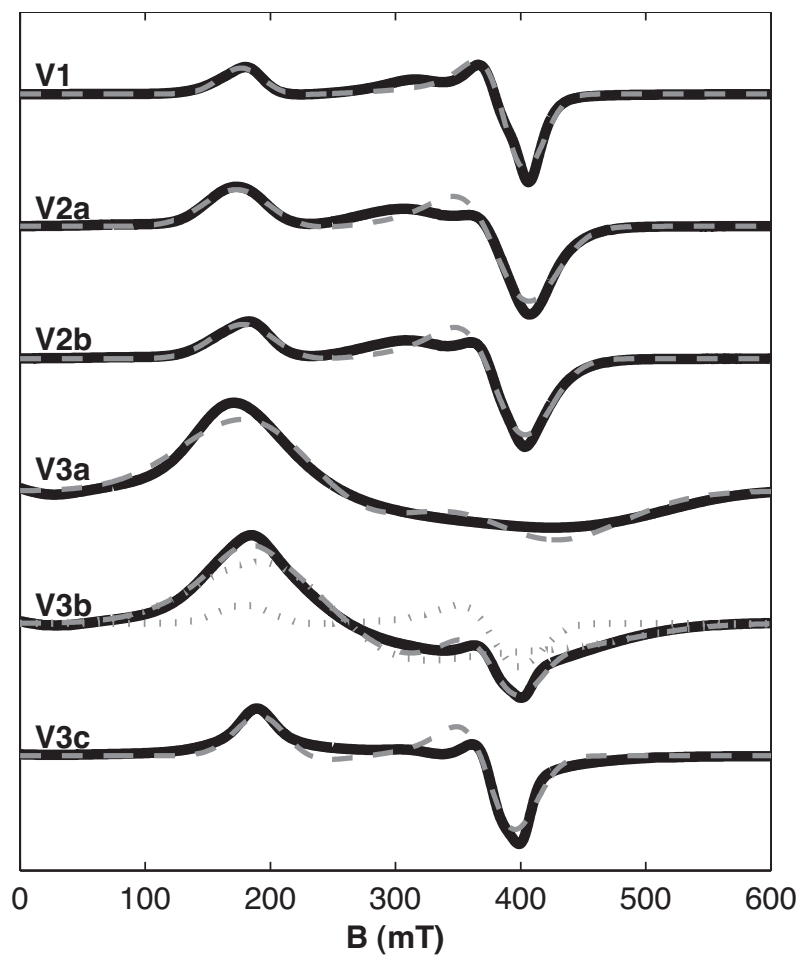

Figure 4. FMR spectra of MV-1. Solid lines show measured spectra, dashed lines show fitted spectra, and dotted lines show the spectra of the individual fit components for two-component fits. 


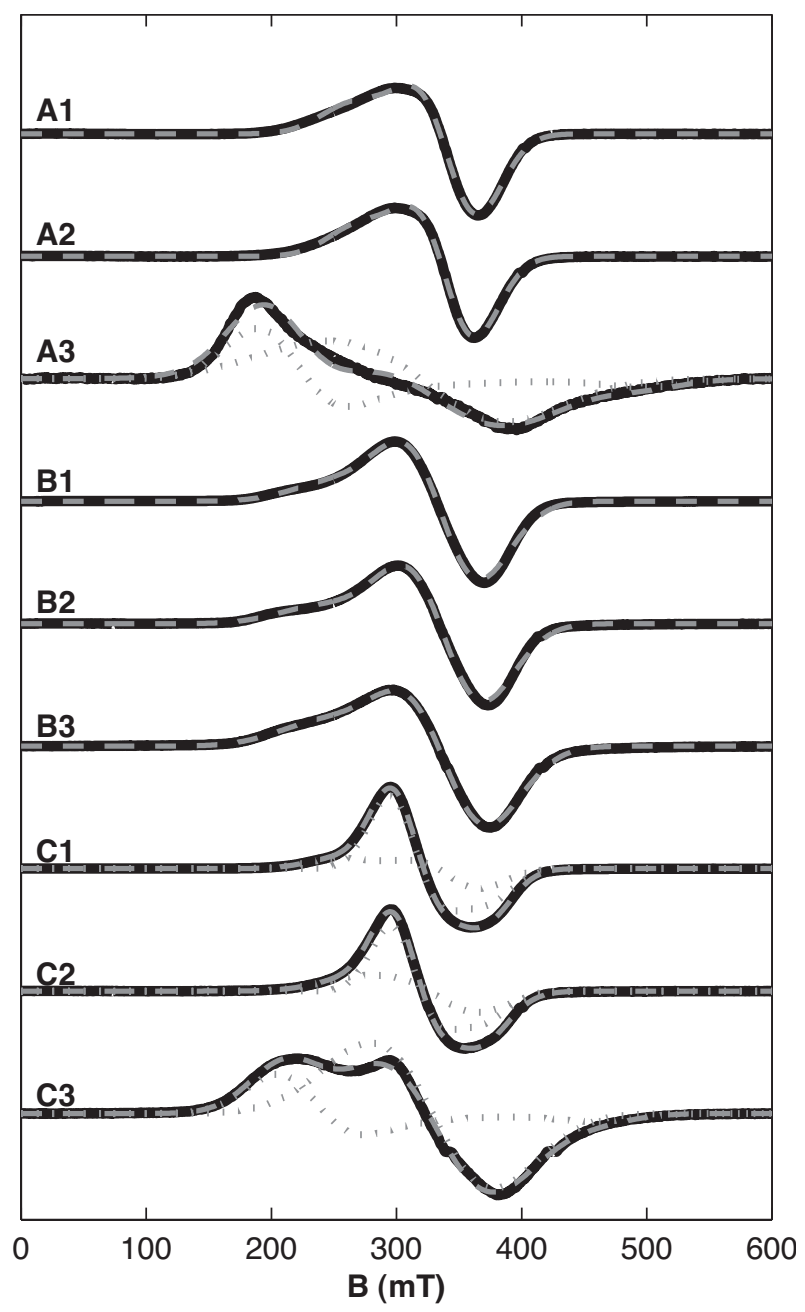

Figure 5. FMR spectra of AMB-1 wild-type (A1-A3), mnm 13 (B1-B3), and mnm 18 (C1-C3). Solid lines show measured spectra, dashed lines show fitted spectra, and dotted lines show the spectra of the individual fit components for two-component fits. 

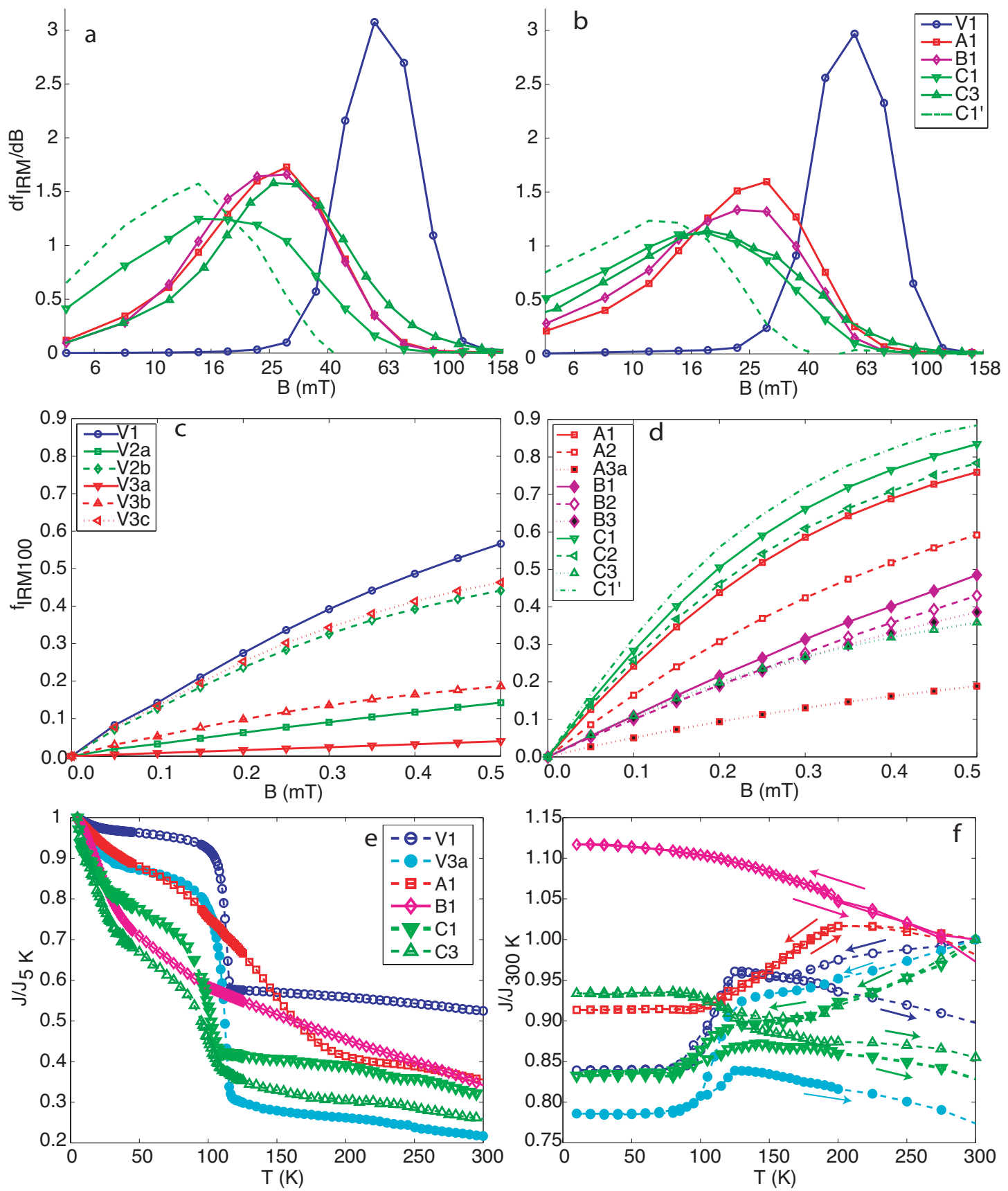

Figure 6. Rock magnetic measures of selected samples. (a-b) Coercivity spectra determined from (a) stepwise IRM acquisition and (b) stepwise AF demagnetization of IRM. The dashed line C1' in (a), (b), and (d) indicates the unmixed isolated particle component of $\mathrm{C} 1$, produced using the proportions of isolated and chain components determined from the FMR spectra to remove the chain component. (c-d) ARM acquisition curves of (c) MV-1 and (d) AMB-1 wild-type and mutants. (ef) Low-temperature demagnetization curves. (e) shows the demagnetization upon warming of a magnetization acquired by saturation at $5 \mathrm{~K}$ of samples cooled in a $3 \mathrm{~T}$ field. Magnetization values are shown normalized to the magnetization at $5 \mathrm{~K}$. (f) shows the demagnetization upon cooling and subsequent warming of a magnetization acquired by saturation at $300 \mathrm{~K}$. Magnetization values are shown normalized to the initial room-temperature magnetization. 

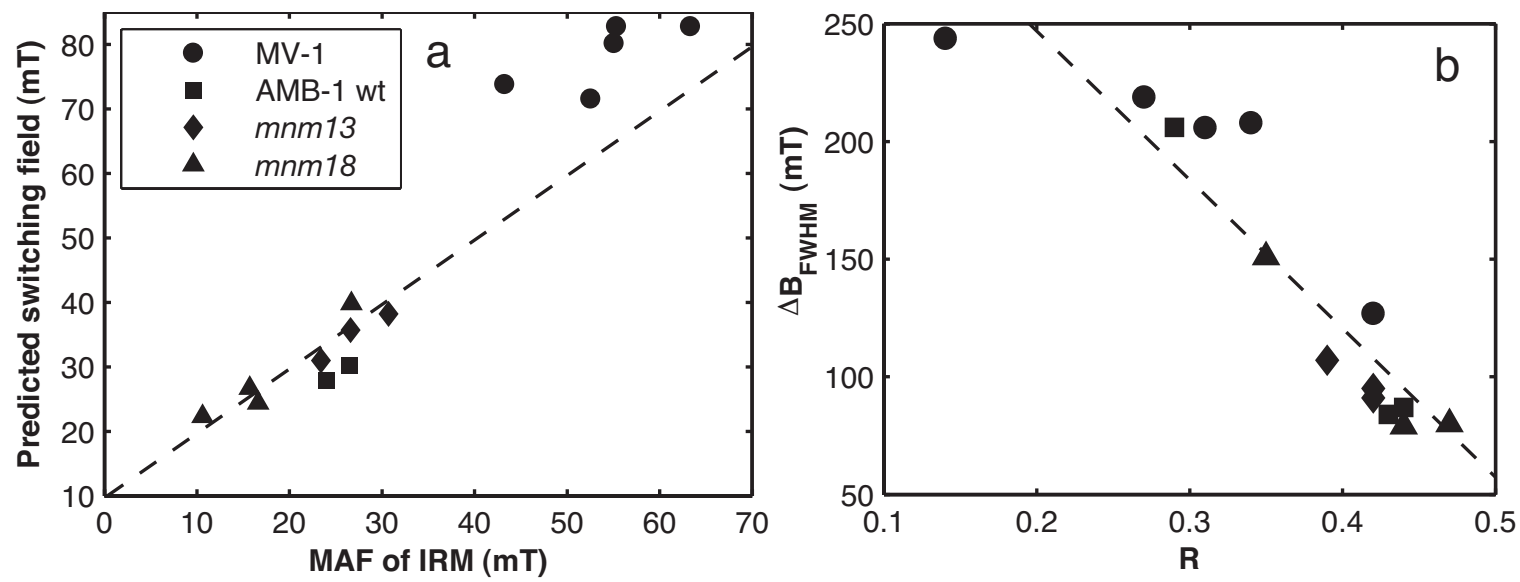

Figure 7. FMR parameters compared to rock magnetic parameters for the samples discussed in this paper. (a) Predicted switching field, determined from the weighted average of of fit components for each sample, plotted against the median acquisition field of IRM. The dashed line represents a line fitted through the points for $m n m 13$ and $m n m 18$ with slope fixed at 1 . The line has a yintercept of $9.7 \mathrm{mT}$ and a coefficient of determination $\mathrm{r}^{2}=0.89$. (b) $\Delta \mathrm{B}_{\mathrm{FWHM}}$ plotted against the Cisowski $R$ parameter, which measures magnetostatic interactions. The dashed line represents a line fitted to all samples and is given by $\Delta \mathrm{B}_{\mathrm{FWHM}}=373 \mathrm{mT}-632 \mathrm{mT} \times \mathrm{R}$. It has a coefficient of determination $\mathrm{r}^{2}=0.84$ 


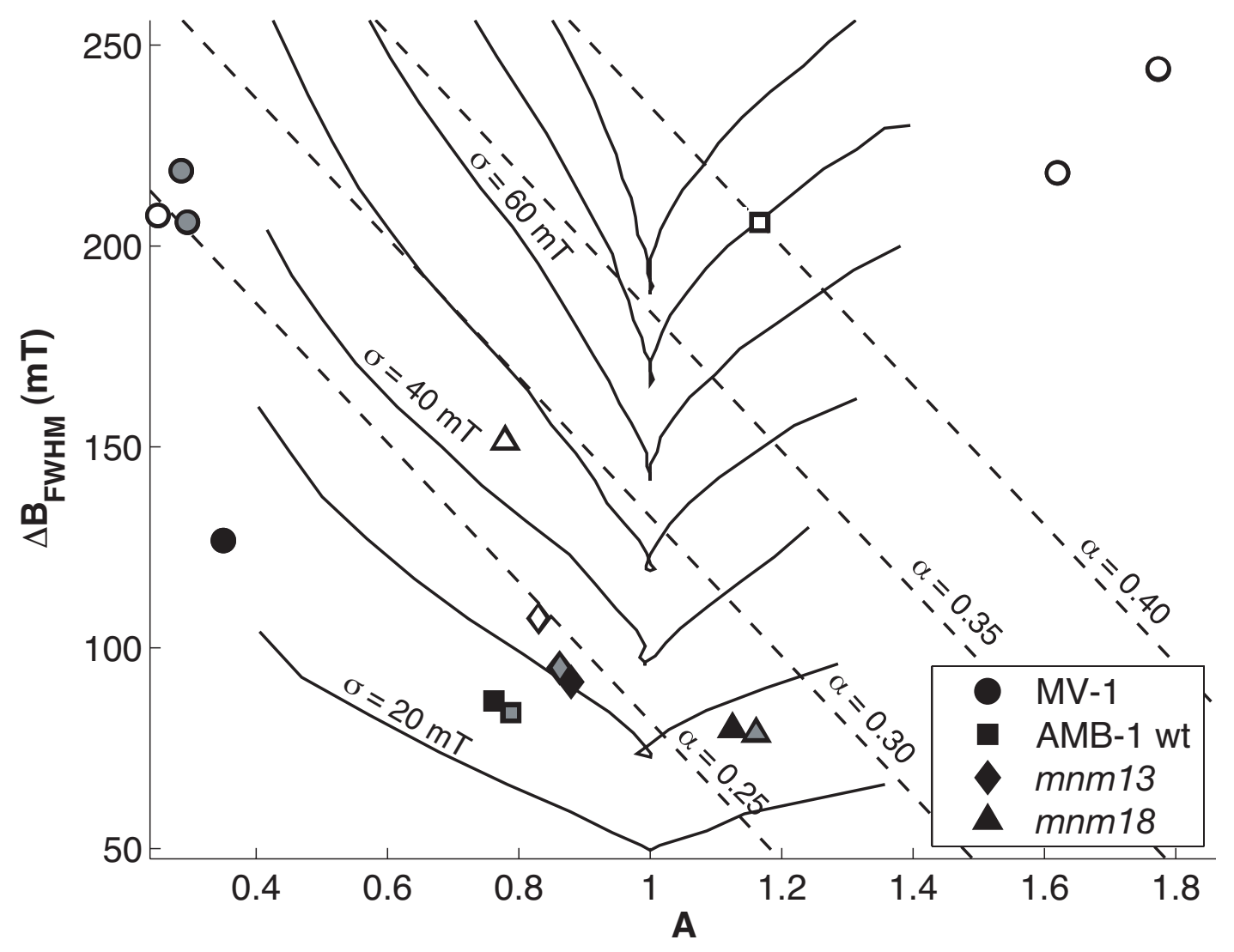

Figure 8. Plot of $\Delta \mathrm{B}_{\mathrm{FwHM}}$ against $\mathrm{A}$ for the samples discussed in this paper. Solid symbols represent untreated samples, symbols with grey interiors represent ultrasonicated samples, and samples with white interiors represented SDS-treated samples. The dilution trend for ultrasonicated MV-1 goes slightly from the upper-left to the bottom-right, while the dilution trend for SDS-treated MV1 goes from right to left. Dashed lines are contours of constant values of $\alpha$. Solid lines represent simulated spectra with fixed Gaussian linewidth $\sigma$ and variable $B_{a n}$. 